\title{
Identifying the neutrino mass spectrum from a supernova neutrino burst
}

\author{
Amol S. Dighe* \\ Theory Division, CERN, CH-1211 Geneva 23, Switzerland \\ Alexei Yu. Smirnov ${ }^{\dagger}$ \\ The Abdus Salam International Center for Theoretical Physics, 34100 Trieste, Italy \\ and Institute of Nuclear Research, RAS, Moscow, Russia \\ (Received 31 January 2000; published 12 July 2000)
}

\begin{abstract}
We study the role that the future detection of the neutrino burst from a galactic supernova can play in the reconstruction of the neutrino mass spectrum. We consider all possible $3 \nu$ mass and flavor spectra which describe the solar and atmospheric neutrino data. For each of these spectra we find the observable effects of the supernova neutrino conversions both in the matter of the star and the Earth. We show that studies of the electron neutrino and antineutrino spectra as well as observations of the neutral current effects from supernova will allow us (i) to identify the solar neutrino solution, (ii) to determine the type of mass hierarchy (normal or inverted) and (iii) to probe the mixing $\left|U_{e 3}\right|^{2}$ to values as low as $10^{-4}-10^{-3}$.
\end{abstract}

PACS number(s): $14.60 . \mathrm{Pq}, 97.60 . \mathrm{Bw}$

\section{INTRODUCTION}

The reconstruction of the neutrino mass and flavor spectrum is one of the fundamental problems of particle physics. It also has important implications for cosmology and astrophysics. Knowledge of neutrino masses and mixing will allow us to clarify the role of neutrinos in the mechanism of star explosions and supernova nucleosynthesis.

With the present data on the atmospheric and solar neutrinos, we are taking the first steps in the reconstruction of the spectrum. The SuperKamiokande (SK) results [1] on atmospheric neutrinos, confirmed by the recent SOUDAN [2] and MACRO [3] data, allow us to claim with a high confidence level that the atmospheric neutrinos oscillate. Moreover, the oscillations are due to neutrino masses and the mixing in vacuum. The data also indicate $\nu_{\mu} \leftrightarrow \nu_{\tau}$ as the dominant mode. All the existing experimental results can be well described in terms of the $\nu_{\mu} \leftrightarrow \nu_{\tau}$ vacuum oscillations with the mass squared difference and the mixing parameters given by [1]

$$
\left|\Delta m_{\text {atm }}^{2}\right|=(1-8) \times 10^{-3} \mathrm{eV}^{2}, \quad \sin ^{2} 2 \theta=0.8-1.0 \text {. }
$$

There is no compelling evidence that the electron neutrinos participate in the oscillations of atmospheric neutrinos. Moreover, the CHOOZ experiment [4] gives an upper bound on the mixing of $\nu_{e}$ with $\Delta m^{2} \sim \Delta m_{\text {atm }}^{2}$ :

$$
\sin ^{2} 2 \theta_{e} \leqslant 0.1 \text { for }\left|\Delta m^{2}\right|>2 \times 10^{-3} \mathrm{eV}^{2} \text {. }
$$

The oscillation interpretation of the atmospheric neutrino data indicates that the solution of the solar neutrino problem is also related to nonzero neutrino masses and mixing. At the moment, however, there are several possible solutions. Moreover, various sorts of data-spectral distortions, day-

\footnotetext{
*Email address: amol.dighe@cern.ch

†Email address: smirnov@ictp.trieste.it
}

night effects, seasonal variations-favor different possible solutions. A good description of all the existing data can be obtained by [5]

(1) the small mixing angle (SMA) Mikheyev-SmirnovWolfenstein (MSW) solution

$$
\Delta m_{\odot}^{2}=(4-10) \times 10^{-6} \mathrm{eV}^{2}, \quad \sin ^{2} 2 \theta_{\odot}=(2-10) \times 10^{-3},
$$

(2) the large mixing angle (LMA) MSW solution

$$
\Delta m_{\odot}^{2}=(1-10) \times 10^{-5} \mathrm{eV}^{2}, \quad \sin ^{2} 2 \theta_{\odot}=0.7-0.95,
$$

(3) the vacuum oscillation (VO) solution

$$
\begin{aligned}
\Delta m_{\odot}^{2} & = \begin{cases}(4-6) \times 10^{-10} & \mathrm{eV}^{2}, \\
(6-8) \times 10^{-11} & \mathrm{eV}^{2},\end{cases} \\
\sin ^{2} 2 \theta_{\odot} & =0.8-1.0 .
\end{aligned}
$$

Some other possibilities are also not excluded-e.g. the LOW (low mass, low probability) MSW solution with $\Delta m^{2}$ $\sim(0.5-2) \times 10^{-7} \mathrm{eV}^{2}$ and $\sin ^{2} 2 \theta_{\odot}=0.9-1.0$ (see $[5,6]$ ). Results from future experiments with existing and new detectors will remove this ambiguity, thus identifying the correct solution to the solar neutrino problem.

Further evidence for neutrino oscillations follows from the Liquid Scintillation Neutrino Detector (LSND) results [7], which are not confirmed, but also not excluded by the KARMEN experiment [8]. The LSND results cannot be reconciled with solutions of the atmospheric and solar neutrino problems in the context of only three known neutrinos, thus requiring the introduction of sterile neutrinos [9]. In this paper, we shall consider only the mixing between the known three neutrinos (spectra with sterile neutrinos will be discussed elsewhere).

The atmospheric and solar neutrino results $[1-3,5]$ as well as the existing bounds from the other oscillation experiments and the $\beta \beta_{0 \nu}$ searches lead to several possible spectra of neutrino masses and mixing. The ambiguity is related to (i) 
the unidentified solution of the solar neutrino problem, (ii) the unknown mixing of $\nu_{e}$ in the third mass eigenstate which is described by the matrix element $U_{e 3}$ and (iii) the type of hierarchy (normal or inverted) which is related to the mass of the third mass eigenstate (whether it is the lightest or the heaviest one). The absolute scale of the mass is also unknown; however, this cannot be established from the oscillation phenomena.

In this paper, we reconsider the effects of oscillations on supernova neutrinos. With the existing data on neutrino masses and mixing, we can sharpen the predictions of the oscillation effects in the supernova neutrinos. On the other hand, we clarify the extent to which studies of supernova neutrinos can contribute to the reconstruction of the neutrino mass and flavor spectrum. We will show that the three ambiguities mentioned above can in principle be resolved by supernova data.

The effects of neutrino mixing on the neutrino fluxes from the supernova have been extensively discussed in the context of $2 \nu$ mixing. For a wide range of mixing parameters $\left(\Delta m^{2} \lesssim 10^{4} \mathrm{eV}^{2}\right)$, the neutrinos encounter their MSW resonance densities inside the star; hence the studies of resonant neutrino conversions inside the star [10-12] are crucial. For very low values of $\Delta m^{2}$ (e.g. $\Delta m^{2} \leqslant 10^{-14} \mathrm{eV}^{2}$ ), the vacuum oscillations on the way from the star to the Earth need to be taken into account $[13,14]$. In the presence of a strong magnetic field, spin-flip effects become important [15]: the spin-flavor precession [16] and resonant spin-flavor conversions [17] may affect the observed neutrino fluxes. If sterile neutrinos are involved in the neutrino conversions, they may enable $r$-process nucleosynthesis [18].

The effects of the neutrino conversions can be observed through, e.g. (i) the disappearance (partial or complete) of the neutronization peak, (ii) the interchange of original spectra and the appearance of a hard $\nu_{e}$ spectrum, (iii) distortions of the $\nu_{e}$ energy spectrum, (iv) modification of the $\bar{\nu}_{e}$ spectrum (in particular, the effects of large lepton mixing on the $\bar{\nu}_{e}$ spectrum have been extensively studied [19]), and (v) Earth matter effects. The observation of the neutrino burst from SN 1987A [20] has already given bounds on the large mixing of active neutrinos $[11,12,19-22])$ and on the mixing of $\nu_{e}$ with sterile neutrinos [23].

The main features of transitions of supernova neutrinos in the case of $3 \nu$ mixing $[22,24-30]$ can be understood in terms of the $2 \nu$ mixing. The system has two resonances. ${ }^{1}$ Under the assumptions of mass hierarchy and smallness of mixing, the dynamics of the two level crossings splits. As a result, the factorization of probabilities occurs $[24,25]$. In the presence of sterile neutrinos, multi-level conversions take place $[28,31]$, which may be interpreted in terms of the constituent $2 \nu$ conversions for small mixing angles and $\Delta m^{2}$ hierarchy [9].

\footnotetext{
${ }^{1}$ The radiative corrections to $m_{\nu_{\mu}}$ and $m_{\nu_{\tau}}$ imply the existence of one more resonance between the two non-electron neutrinos, but since the two non-electron neutrinos cannot be distinguished at the detector, the conversions between them do not affect the observations. See Sec. II F.
}

In this paper, we study the conversions of supernova neutrinos in the $3 \nu$ context, taking into account recent results on the neutrino masses and mixing. We consider the effects for all possible schemes of neutrino masses and mixing which explain the atmospheric and solar neutrino data. For each scheme, we find the modifications of (i) the neutronization peak, (ii) the $\nu_{e}$ energy spectrum and (iii) the $\bar{\nu}_{e}$ energy spectrum, which can be observed directly. We also determine the spectrum of the non-electron neutrinos which can in principle be studied by neutral current interactions in reactions with different energy thresholds.

The paper is organized as follows. In Sec. II we describe the features of initial neutrino fluxes from the supernova and the dynamics of neutrino conversion on their way out to the surface of the star. In Sec. III, we derive general expressions for the transition probabilities for the schemes with normal mass hierarchy. We also calculate the Earth matter effects on the neutrino spectra. In Sec. IV, we find the final neutrino spectra at detectors for the schemes with the normal mass hierarchy. In Sec. V, we perform similar studies for the schemes with the inverted mass hierarchy. In Sec. VI, we discuss the observable signals and the signatures of various mixing schemes In Sec. VII, comparing results for various schemes we conclude about the possibility to discriminate the schemes by future observations of neutrino bursts from a galactic supernova.

\section{MASS SPECTRA, FLUXES AND DYNAMICS OF CONVERSION}

In this section, the generic properties of the initial neutrino fluxes will be summarized. We identify the neutrino mass and mixing parameters relevant for the supernova neutrino conversions, and consider main aspects of dynamics of neutrino conversion inside the star: the transition regions, the factorization of dynamics and adiabaticity. Finally, we construct the level crossing schemes for the normal and the inverted mass hierarchy.

\section{A. Neutrino fluxes}

In what follows we will summarize the generic features of the original fluxes which do not depend on the model and the parameters of the star. The deviations from these features will testify for neutrino conversions.

(1) Flavor of the neutronization peak. During first few milliseconds of the neutrino burst from a supernova, the signal is expected to be dominated by the $\nu_{e}$, which are produced by the electron capture on protons and nuclei while the shock wave passes through the neutrinosphere [32]. Since the original flux is $\nu_{e}$, the final observed fluxes give a direct measurement of the extent of conversion of $\nu_{e}$ into the other neutrino species.

(2) Inequalities of average energies of the spectra. Since $\nu_{e}$ interact more strongly with matter than the other species, their effective neutrinosphere is outside the neutrinospheres of the other species and hence they have a lower average energy than $\bar{\nu}_{e}$ and $\nu_{x}$. The $\bar{\nu}_{e}$ also interact via charged current, but the cross section is smaller, so their average 


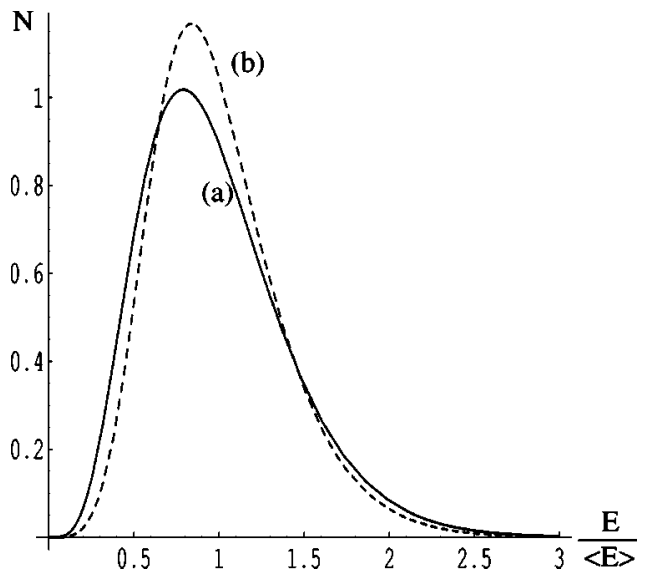

FIG. 1. The number of $\nu-N$ charged current events for (a) a thermal spectrum $(T=3, \eta=0)$, (b) a "pinched" $\operatorname{spectrum~}(T$ $=3, \eta=3$ ). $T$ and $\eta$ are the parameters as in Eq. (7). Both the energy resolution function and the lower energy threshold effects have been taken into account. The spectra are normalized to have equal areas.

energy is more than that of the $\nu_{e}$, but less than that of $\nu_{x}$ :

$$
\left\langle E_{\nu_{e}}^{0}\right\rangle<\left\langle E_{\nu_{e}}^{0}\right\rangle<\left\langle E_{\nu_{x}}^{0}\right\rangle \text {. }
$$

Here the superscript " 0 " refers to the original spectra. The neutrino conversion changes inequalities (6).

(3) The pinched spectra during the cooling stage. Let $F_{e}^{0}$ and $F_{\bar{e}}^{0}$ be the original fluxes of $\nu_{e}$ and $\bar{\nu}_{e}$ respectively, produced during the cooling stage. The "non-electron" neutrinos $\left(\nu_{\mu}, \nu_{\tau}, \bar{\nu}_{\mu}, \bar{\nu}_{\tau}\right)$ have the same neutral current interactions inside the supernova, and their original fluxes are expected to be approximately equal. ${ }^{2}$ In what follows we will neglect the difference of fluxes. We will denote these four neutrino species collectively as $\nu_{x}$ and the original flux of each of them as $F_{x}^{0}$.

The spectra of neutrinos from the cooling stage are not exactly thermal. Since the neutrino interaction cross section increases with energy, even for the same species of neutrinos, the effective neutrinosphere radius increases with increasing energies. Then, even if the neutrinos at their respective neutrinospheres are in thermal equilibrium with the matter, the spectrum gets "pinched," i.e. depleted at the higher as well as the lower end of energies in comparison with thermal spectrum. (See Fig. 1 for an illustration.) It can be shown that pinching of the instantaneous spectrum is the consequence of the following two facts: (i) the temperature inside the supernova decreases with increasing radius and (ii) the density decreases faster than $1 / r$ [34]. The pinching of the instantaneous spectrum can be extended to the pinching of the time-integrated spectrum as long as the time variation

\footnotetext{
${ }^{2}$ The presence of real muons in the central part of the star leads to a nonzero chemical potential of the muon neutrinos and hence to a difference of fluxes [33]. However, in the neutrinosphere with $T$ $\approx 6-8 \mathrm{MeV}$, the concentration of muons is smaller than $1 \%$.
}

of the average energy of the spectrum is small. Numerical simulations of the neutrino spectra confirm the pinching of spectra $[35,36]$.

One way to parametrize the pinched neutrino spectra is to introduce an effective temperature $T_{i}$ and an effective degeneracy parameter $\eta_{i}$ (which has the same sign for neutrinos and antineutrinos and cannot be considered as the chemical potential) in the Fermi-Dirac thermal spectrum for each species $i$ :

$$
F_{i}^{0}(E) \propto \frac{E^{2}}{\operatorname{Exp}\left(E / T_{i}-\eta_{i}\right)+1} .
$$

For a pinched spectrum, $\eta_{i}>0$. The value of $\eta_{i}$ is the same for all $\nu_{x}$ species (neutrinos as well as antineutrinos, since they have the same interactions), and are in general different from $\eta_{e}$ or $\eta_{e}^{-}$. The value of $\eta_{i}$ need not be constant throughout the cooling stage. Typically,

$$
T_{e} \approx 3-4 \mathrm{MeV}, \quad T_{e}^{-} \approx 5-6 \mathrm{MeV}, \quad T_{x} \approx 7-9 \mathrm{MeV} .
$$

The typical values of $\eta_{i}$ are $[36,37]$

$$
\eta_{e} \approx 3-5, \quad \eta_{e}^{-\approx 2.0-2.5, \quad \eta_{x} \approx 0-2 .}
$$

Notice that the strongest pinching is expected for the $\nu_{e}$ spectrum. These values, however, are model dependent and we shall use them only as a guide.

In what follows, we shall calculate the fluxes of electron neutrinos $F_{e}$, electron antineutrinos, $F_{e}^{-}$, and the total flux of the non-electron neutrinos, " $4 F_{x}$," at the Earth detectors.

\section{B. Neutrino mass spectra}

We consider the system of three active neutrinos $\vec{\nu}_{f}$ $\equiv\left(\nu_{e}, \nu_{\mu}, \nu_{\tau}\right)$ mixed in vacuum, such that

$$
\vec{\nu}_{f}=U \vec{\nu}
$$

where $\vec{\nu} \equiv\left(\nu_{1}, \nu_{2}, \nu_{3}\right)$ is the vector of mass eigenstates and

$$
U \equiv\left\|U_{f i}\right\|, \quad f=e, \mu, \tau, \quad i=1,2,3,
$$

is the mixing matrix. We take

$$
\Delta m_{32}^{2} \equiv m_{3}^{2}-m_{2}^{2}=\Delta m_{a t m}^{2},
$$

so that the oscillations driven by $\Delta m_{23}^{2}$ solve the atmospheric neutrino problem. We identify

$$
\Delta m_{21}^{2} \equiv m_{2}^{2}-m_{1}^{2}=\Delta m_{\odot}^{2},
$$

where $\Delta m_{\odot}^{2}$ is in one of the regions (SMA, LMA or VO) implied by the solar neutrino data [Eqs. (3),(4),(5)].

The key features of the spectra which play an important role in the applications to the supernova neutrinos are

(1) the hierarchy of $\Delta m^{2}$,

$$
\left|\Delta m_{32}^{2}\right| \approx\left|\Delta m_{31}^{2}\right| \gg\left|\Delta m_{21}^{2}\right|
$$

(2) the upper bound on $\Delta m^{2}$, 


$$
\left|\Delta m_{i j}^{2}\right| \lesssim 10^{-2} \mathrm{eV}^{2}
$$

Since $\nu_{\mu}$ and $\nu_{\tau}$ are indistinguishable in the supernova neutrino studies, the neutrino transitions are determined by the mixings of the electron neutrino only, i.e. by the elements $U_{e i}$ (11) (see Sec. III for more details). Moreover, the three $U_{e i}$ 's are related by the unitarity condition $\Sigma\left|U_{e i}\right|^{2}=1$, so that only two mixing elements are relevant, and one can use $\left|U_{e 2}\right|$ and $\left|U_{e 3}\right|$.

The element $U_{e 3}$ is small, as mentioned in the Introduction. If $\left|U_{e 3}\right|^{2} \ll 1$, then $U_{e 2}$ (and therefore, $U_{e 1}$ ) can be found from the solar neutrino data:

$$
4\left|U_{e 2}\right|^{2}\left|U_{e 1}\right|^{2} \approx 4\left|U_{e 2}\right|^{2}\left(1-\left|U_{e 2}\right|^{2}\right) \approx \sin ^{2} 2 \theta_{\odot},
$$

where $\theta_{\odot}$ is the mixing angle determined in the $2 \nu$ analysis of the solar neutrino data.

The system is then determined by two pairs of parameters $\left(\Delta m_{i}^{2}, \sin ^{2} 2 \theta_{i}\right), i=L, H$, where

$$
\begin{aligned}
&\left(\Delta m_{L}^{2}, \sin ^{2} 2 \theta_{L}\right) \cong\left(\Delta m_{\odot}^{2}, \sin ^{2} 2 \theta_{\odot}\right) \\
&\left(\Delta m_{H}^{2}, \sin ^{2} 2 \theta_{H}\right) \cong\left(\Delta m_{a t m}^{2}, 4\left|U_{e 3}\right|^{2}\right) .
\end{aligned}
$$

Correspondingly, it can then be described by two points in the $\left(\Delta m^{2}, \sin ^{2} 2 \theta\right)$ plane.

The current oscillation data do not determine the mass and flavor spectrum completely. As we have already mentioned in the Introduction, the uncertainty is related to

(1) The discrete ambiguity in the solution of the solar neutrino problem: The data favor three solutions indicated in the introduction (SMA, LMA and VO), and some other solutions (e.g. the low $\Delta m^{2}$ MSW solution [5,6], the trimaximal mixing solution [38]), although disfavored, are not excluded. The future solar neutrino experiments will remove this ambiguity and sharpen the determination of the oscillation parameters.

(2) The ambiguity in the sign of $\Delta m_{32}^{2}$ (and $\Delta m_{31}^{2}$ ): this determines the type of neutrino mass hierarchy. We refer to the case

$$
\Delta m_{32}^{2}>0, \quad \text { i.e. } m_{3}>m_{2}, m_{1},
$$

as the spectrum with normal mass hierarchy and to the case

$$
\Delta m_{32}^{2}<0, \quad \text { i.e. } m_{2}, m_{1}>m_{3} \text {, }
$$

as the spectrum with inverted mass hierarchy.

The key difference between these two hierarchies is that, in the normal hierarchy, the small $U_{e 3}$-admixture of $\nu_{e}$ is in the heaviest state whereas in the inverted hierarchy, this admixture is in the lightest state.

The type of hierarchy can in principle be established in future studies of atmospheric neutrinos. If the effects of $\nu_{e}$ oscillations will be observed and the sign of the charged lepton produced by the atmospheric neutrinos will be identified in the future experiments, the studies of matter effects in the neutrino and antineutrino channels will allow to establish the sign of $\Delta m^{2}$. The sign can also be found from the studies of matter effects in the future long baseline experiments, in particular, with neutrino factories.

(3) The value of $U_{e 3}$ is unknown. In principle, future atmospheric neutrino experiments and the long baseline experiments will be able to measure or further restrict $U_{e 3}$.

(4) In the case of the SMA and LMA solutions, $\Delta m_{21}^{2}$ $>0$. But the sign of $\Delta m_{21}^{2}$ is undetermined in the VO solution. The feasibility of resolving this ambiguity has been recently discussed in [39].

Summarizing, the ambiguity in the present analysis is related to the solution of the solar neutrino problem, the type of hierarchy and the value of $U_{e 3}$. The first two ambiguities lead to six possible schemes of neutrino masses and mixing. Within each scheme, the predictions depend on the value of $U_{e 3}$.

\section{Neutrino conversion regions}

In supernova, the transitions occur mainly in the resonance layers, where the density varies between ( $\rho_{\text {res }}$ $\left.-\Delta \rho_{\text {res }}\right)$ and $\left(\rho_{\text {res }}+\Delta \rho_{\text {res }}\right)$. Here $\rho_{\text {res }}$ is the resonance density:

$$
\rho_{\text {res }} \approx \frac{1}{2 \sqrt{2} G_{F}} \frac{\Delta m^{2}}{E} \frac{m_{N}}{Y_{e}} \cos 2 \theta,
$$

$G_{F}$ is the Fermi constant, $m_{N}$ is the mass of the nucleon, $E$ is the neutrino energy and $Y_{e}$ is the electron fraction-the number of electrons per nucleon. ${ }^{3}$ For small vacuum mixing, the width of the resonance layer equals

$$
2 \Delta \rho_{\text {res }} \approx 2 \rho_{\text {res }} \tan 2 \theta .
$$

Using Eq. (20), the resonance matter density can be written as

$$
\rho_{\text {res }} \sim 1.4 \times 10^{6} \mathrm{~g} / \mathrm{cc}\left(\frac{\Delta m^{2}}{1 \mathrm{eV}^{2}}\right)\left(\frac{10 \mathrm{MeV}}{E}\right)\left(\frac{0.5}{Y_{e}}\right) \cos 2 \theta .
$$

There are two resonance layers. The layer at higher densities ( $H$-resonance layer), which corresponds to $\Delta m_{a t m}^{2}$, is at

$$
\rho_{H} \sim 10^{3}-10^{4} \mathrm{~g} / \mathrm{cc}
$$

and the layer at lower densities ( $L$-resonance layer), characterized by $\Delta m_{\odot}^{2}$, is at

$$
\rho_{L}=\left\{\begin{array}{rlc}
5-15 & \mathrm{~g} / \mathrm{cc} & \text { for SMA, } \\
10-30 & \mathrm{~g} / \mathrm{cc} & \text { for LMA, } \\
<10^{-4} & \mathrm{~g} / \mathrm{cc} & \text { for VO }
\end{array}\right.
$$

\footnotetext{
${ }^{3}$ It is worthwhile to introduce the resonance density for "VO" parameters too, although the dominating effect could be the vacuum oscillations. We shall consider the limit of $\sin ^{2} 2 \theta \rightarrow 1$ as a special case.
} 
The regions where the neutrino transitions occur are thus far outside the core of the star-in the outer layers of the mantle. Therefore

(i) the transitions do not influence the dynamics of collapse or the cooling of the core;

(ii) the $r$-processes, which occur at $\rho \geq 10^{5}-10^{6} \mathrm{~g} / \mathrm{cc}$, are also not affected;

(iii) the shock wave does not influence the neutrino conversions [indeed, during the time of cooling by neutrino emission $(t \sim 10 \mathrm{sec})$, the shock wave can only reach layers with densities $\left.\rho \gtrsim 10^{6} \mathrm{~g} / \mathrm{cc}[40,41]\right]$;

(iv) the density profile encountered by the neutrinos during their resonant conversions is almost static, and the same as that of the progenitor star.

In the region with densities $\gtrsim 1 \mathrm{~g} / \mathrm{cc}$, the electron fraction is almost constant and the density profile can be approximated by $[12,36,42]$

$$
\rho Y_{e} \approx 2 \times 10^{4} \mathrm{~g} / \mathrm{cc}\left(\frac{r}{10^{9} \mathrm{~cm}}\right)^{-3}, \text { for } \rho \gtrsim 1 \mathrm{~g} / \mathrm{cc} .
$$

For $\rho \lesssim 1 \mathrm{~g} / \mathrm{cc}$, the fraction of hydrogen increases and $Y_{e}$ becomes larger than 0.5 . The exact shape of the density profile depends on the details of the composition of the star.

\section{Factorization of the dynamics}

The hierarchy of $\Delta m^{2}$, and therefore the hierarchy of the densities of the resonance layers, leads to the "factorization" of the dynamics of conversion: the transitions in the two resonance layers can be considered independently and each transition is reduced to a two neutrino problem. Indeed, in the $H$-resonance region, the mixing $U_{e 2}^{m}$ associated with $\Delta m_{\odot}^{2}$ is suppressed by matter. The suppression factor is

$$
\frac{U_{e 2}^{m}}{U_{e 2}} \sim \frac{\rho_{L}}{\rho_{H}} \lesssim 10^{-2} .
$$

Correspondingly, the effects driven by $\Delta m_{\odot}^{2}$ are suppressed by more than two orders of magnitude.

In the $L$-resonance region, the mixing associated with $\Delta m_{\text {atm }}^{2}$ coincides with the vacuum mixing: $U_{e 3}^{m} \approx U_{e 3}$. The matter corrections are strongly suppressed:

$$
U_{e 3}^{m}=U_{e 3}[1+\mathcal{O}(\xi)], \quad \xi \approx \frac{\rho_{L}}{\rho_{H}} \lesssim 10^{-2} .
$$

That is, the mixing associated with $\Delta m_{\text {atm }}^{2}$ is almost constant, and therefore the level $\nu_{3}$ practically does not participate in the dynamics. By an appropriate redefinition of the fields, the problem can be reduced to a two state problem. The state $\nu_{3}$ decouples from the rest of the system, producing just an averaged oscillation effect [26].

If the mixing $U_{e 3}$ is very small, the resulting survival probability of $\nu_{e}\left(\bar{\nu}_{e}\right)$ is also factorized [24,25]:

$$
\begin{aligned}
{\left[P\left(\nu_{e} \rightarrow \nu_{e}\right)\right]_{\text {total }}=} & {\left[P\left(\nu_{e} \rightarrow \nu_{e}\right)\right]_{H} \times\left[P\left(\nu_{e} \rightarrow \nu_{e}\right)\right]_{L} } \\
& +\mathcal{O}\left(\left|U_{e 3}\right|^{2}\right),
\end{aligned}
$$

and similarly for $\bar{\nu}_{e}$.

\section{E. Adiabatic, non-adiabatic and transition regions}

The dynamics of transitions in each resonance layer is determined by the adiabaticity parameter $\gamma[43,44]$ :

$$
\gamma \equiv \frac{\Delta m^{2}}{2 E} \frac{\sin ^{2} 2 \theta}{\cos 2 \theta} \frac{1}{\left(1 / n_{e}\right)\left(d n_{e} / d r\right)},
$$

such that the "flip" probability - the probability that a neutrino in one matter eigenstate jumps to the other matter eigenstate-is

$$
P_{f}=\exp \left(-\frac{\pi}{2} \gamma\right)
$$

as given by the Landau-Zener formula [43]. Adiabatic conversion corresponds to $\gamma \gg 1$, i.e. to a very small flip probability. ${ }^{4}$

Let us consider the density profile of the form

$$
\rho=A / r^{n},
$$

where $A$ is the proportionality constant. From Eq. (29), we get the adiabaticity parameter for this profile as

$$
\gamma=\frac{1}{2 n}\left(\frac{\Delta m^{2}}{E}\right)^{1-1 / n} \frac{\sin ^{2} 2 \theta}{(\cos 2 \theta)^{1+1 / n}}\left(\frac{2 \sqrt{2} G_{F} Y_{e}}{m_{N}} A\right)^{1 / n} .
$$

Here we have used the resonance condition to express $r$ through the oscillation parameters. Note that the dependence of $\gamma$ on the absolute scale of density, $A$, is rather weak:

$$
\gamma \propto A^{1 / n}
$$

For $n=3$, the change of $A$ by one order of magnitude leads to the change of $\gamma$ by a factor of 2 .

For a fixed density scale $A$, the value of $\gamma$ depends on the power index $n$ as

\footnotetext{
${ }^{4}$ The Landau-Zener formula is valid for a linear variation of density in the resonance region and a small mixing angle $\theta$. For an arbitrary density distribution and mixing angle, the Landau-Zener formula (30) gets modified to [45]

$$
P_{f}=\frac{\exp \left(-\frac{\pi}{2} \gamma F\right)-\exp \left(-\frac{\pi}{2} \gamma F / \sin ^{2} \theta\right)}{1-\exp \left(-\frac{\pi}{2} \gamma F / \sin ^{2} \theta\right)},
$$

where $F$ is a function of the density profile and the mixing angle. The adiabaticity condition is, approximately, $\gamma F \gg 1$. At small mixing angles and linear density variation in the resonance region, $F$ $\approx 1$ and Eq. (31) reduces to Eq. (30).
} 


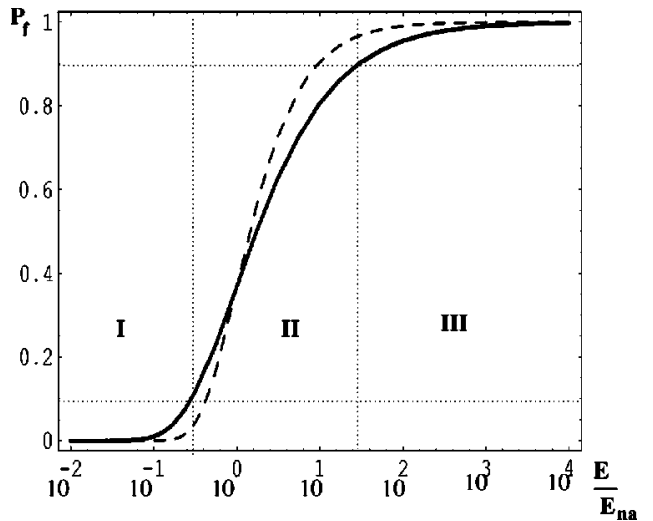

FIG. 2. The energy dependence of $P_{f}$ on $E / E_{n a}$. The solid line is for the density profile $\rho \sim r^{-3}$, whereas the dashed line is for the density profile $\rho \sim e^{-r}$.

$$
\gamma \propto 1 / n
$$

so that a variation of $n$ between 2 and 4 leads to the variation in $\gamma$ by a factor of 2 .

In turn, the uncertainty of a factor of 2 in $\gamma$ is equivalent to the change of $\sin ^{2} 2 \theta$ by a factor of 2 (at small values of $\theta$ ) or the change of $\Delta m^{2}$ by a factor of $2^{n /(n-1)} \sim 3-4$.

The lines of equal $\gamma$ (and therefore, equal $P_{f}$ ) on the $\left(\Delta m^{2}-\sin ^{2} 2 \theta\right)$ plot are determined by

$$
\left(\Delta m^{2}\right)^{1-1 / n} \sin ^{2} 2 \theta=\mathrm{const}
$$

for small $\theta$.

For $n=3$ which will be used in the further calculations, we get

$$
\Delta m^{2}=\text { const } / \sin ^{3} 2 \theta .
$$

From Eqs. (30) and (33), the flip probability as a function of energy can be written as [27]

$$
P_{f}=\exp \left[-\left(\frac{E_{n a}}{E}\right)^{2 / 3}\right],
$$

where

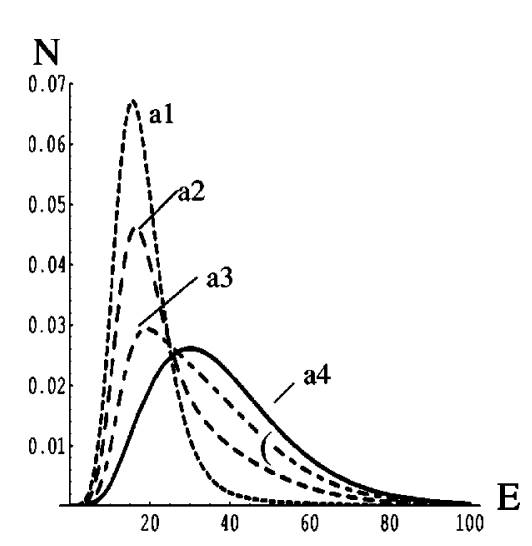

(a)

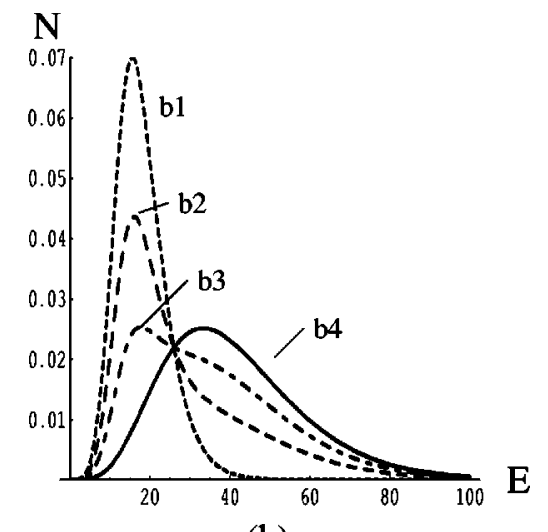

(b)

033007-6

$$
E_{n a}=\left(\frac{\pi}{12}\right)^{3 / 2} \frac{\Delta m^{2} \sin ^{3} 2 \theta}{\cos ^{2} 2 \theta}\left(\frac{2 \sqrt{2} G_{F} Y_{e}}{m_{N}} A\right)^{1 / 2} .
$$

The dependence of $P_{f}$ on $E / E_{n a}$ is shown in Fig. 2. One can divide the whole range of energy into three parts:

For $E / E_{n a}<10^{-1}$ (region I), we get $P_{f} \approx 0$. In this range, pure adiabatic conversion occurs.

For $E / E_{n a}>10^{2}$ (region III), the flip probability is close to 1 , which corresponds to a strong violation of adiabaticity.

In the transition region $E / E_{n a}=10^{-1}-10^{2}$ (region II), $P_{f}$ increases with the neutrino energy. This region spans almost three orders of magnitude in energy, which is substantially larger than the range of energies in the neutrino spectrum. Notice that for the exponential density distribution (which is the case inside the sun), the transition region is narrower (about two orders of magnitude) and correspondingly, the energy dependence in this region is stronger.

The observable part of the supernova neutrino spectrum lies mainly between the energies of 5 and $50 \mathrm{MeV}$; i.e., it spans about one order of magnitude. If the spectrum is in region I, completely adiabatic conversion occurs for the whole spectrum. In region II, the conversion depends on energy; however, the dependence is not strong over the relevant range of energies. The average energies of any two neutrino species differ by a factor of less than 3 , and for $\Delta E / E \sim 3$, the variation in the flip probability is $\Delta P_{f} \leqslant 0.2$ (from Fig. 2). In the first approximation, the final spectrum can then be characterized by an average or effective flip probability. This is illustrated in Fig. 3: the spectra of the number of events taking into account the energy dependence of $P_{f}$ are shown in (a) and the spectra with an effective flip probability $\left\langle P_{f}\right\rangle$ are shown in (b). It may be observed that the spectra with an appropriate value of $\left\langle P_{f}\right\rangle$ can mimic the features of the actual spectra. This is particularly true given the theoretical uncertainties in the shapes of the original spectra.

In Fig. 4, we show the contours of equal flip probability $P_{f}$, Eq. (31), in the $\left(\Delta m^{2}-\sin ^{2} 2 \theta\right)$ plot for two different energies on the borders of the observable spectrum. We also show the parameter ranges which explain the solar and atmospheric neutrino data. The dark band (the "atmospheric neutrino band') corresponds to the allowed range of $\Delta m_{31}^{2}$. The rightmost part of this band is excluded by the $\mathrm{CHOOZ}$
FIG. 3. The number of $\nu_{e}-N$ charged current events, taking into account the energy dependence of the flip probability. The parameters for the original spectra are taken to be $T_{e}=3 \mathrm{MeV}$, $\eta_{e}=3, T_{x}=8 \mathrm{MeV}, \eta_{x}=1$. In (a), the flip probability is $P_{f}=\exp \left[-\left(E_{n a} / E\right)^{2 / 3}\right]$ with (a1) $E_{n a}$ $=0.05$, (a2) $E_{n a}=2$, (a3) $E_{n a}=10$, (a4) $E_{n a}$ $=50 \mathrm{MeV}$. In (b), the effective flip probability $\left\langle P_{f}\right\rangle$ is (b1) 1.0, (b2) 0.85, (b3) 0.6, (b4) 0.0. 


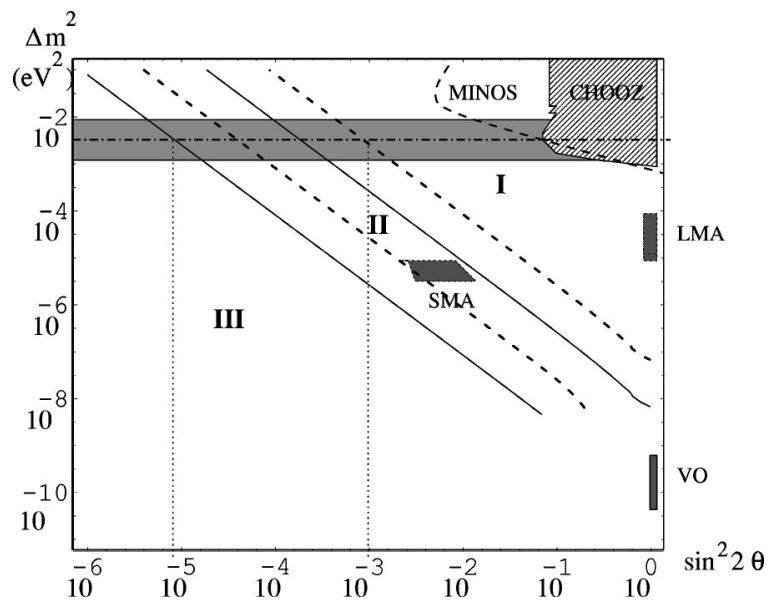

FIG. 4. The contours of equal flip probability $P_{f}$. The solid lines denote the contours of flip probability for a $5 \mathrm{MeV}$ neutrino: the line on the left stands for $P_{f}=0.9$ (highly non-adiabatic transition) and the line on the right stands for $P_{f}=0.1$ (adiabatic transition). The dashed lines represent the corresponding flip probabilities for neutrinos with energy $50 \mathrm{MeV}$. SMA, LMA and VO correspond to the solutions of the solar neutrino anomaly. The two vertical lines indicate the values of $4\left|U_{e 3}\right|^{2}=\sin ^{2} 2 \theta$ lying on the borders of the adiabatic, non-adiabatic and transition regions for $\Delta m^{2}$ corresponding to the best fit value of the atmospheric neutrino solution.

experiment [4]. The range of $\left|U_{e 3}\right|^{2}$ that can be probed by the long baseline experiment MINOS [46] is also shown.

The contours of $P_{f}=0.1$ and $P_{f}=0.9$ divide the plot into three regions, corresponding to the three regions in Fig. 2:

(i) The "adiabatic region" (I) is the region above the contour with $P_{f}=0.1$, where adiabaticity is well satisfied and strong flavor conversions occur.

(ii) The "transition region" (II) is the region between $P_{f}=0.1$ and $P_{f}=0.9$ contours. Here the adiabaticity is partially broken and the transitions are not complete. Moreover, the extent of transitions depends on the energy.

(iii) The "non-adiabatic" region (III) lies below the $P_{f}$ $=0.9$ contour. The neutrino conversions are practically absent.

Since the adiabaticity breaking increases with $E$ and decreases with the increase of $\Delta m^{2}$ and $\sin ^{2} 2 \theta$, the lines of equal $P_{f}$ for $E=50 \mathrm{MeV}$ are shifted to larger $\Delta m^{2}$ and $\sin ^{2} 2 \theta$ relative to the lines for $E=5 \mathrm{MeV}$. The dependence of the contours of equal $P_{f}$ on the density profile is rather weak, as can be seen from the previous discussion. Even with conservative estimates, the borders of the regions have an uncertainty of a factor of 2 in $\sin ^{2} 2 \theta$ for a given value of $\Delta m^{2}$.

As follows from Fig. 4, the LMA solution lies in the adiabatic region $\mathrm{I}^{5}$ The SMA solution is in the transition region. The VO solutions are either in the transition region or

\footnotetext{
${ }^{5}$ The LOW solution also lies in the adiabatic region, so all the results for the LMA scenario are also valid for the LOW scenario.
}

in the non-adiabatic region, which depends essentially on the density profile in the outermost layers of the star $(\rho \leq 1 \mathrm{~g} / \mathrm{cc})$ and the precise value of $\Delta m^{2}$.

As was described in Sec. II B, each neutrino mass and flavor spectrum can be represented by two points (17) in the $\left(\Delta m^{2}, \sin ^{2} 2 \theta\right)$ plot (Fig. 4). One point, corresponding to $\left(\Delta m_{31}^{2}, \sin ^{2} 2 \theta_{e 3}\right)$, should lie in the atmospheric neutrino band, and the other point, corresponding to $\left(\Delta m_{21}^{2}, \sin ^{2} 2 \theta_{e 2}\right)$, should lie in one of the "islands" corresponding to the solutions of the solar neutrino problem. These two points characterize the layers $H$ and $L$, Eqs. (23) and (24) respectively. Let $P_{H}\left(\bar{P}_{H}\right)$ and $P_{L}\left(\bar{P}_{L}\right)$ be the probabilities that the neutrinos (antineutrinos) jump to another matter eigenstate in these two layers. The extent of conversion is determined by the values of these four flip probabilities.

From Fig. 4, we conclude that the $H$ resonance is in the adiabatic range (region I) for

$$
\sin ^{2} 2 \theta_{e 3}=4\left|U_{e 3}\right|^{2} \gtrsim 10^{-3}
$$

and in the transition region (region II) for

$$
\sin ^{2} 2 \theta_{e 3} \sim 10^{-5}-10^{-3} \text {. }
$$

As we shall see in Secs. IV and V, the features of the final spectra depend strongly on the region in which the $H$ resonance lies. According to Eq. (41), the supernova neutrino spectra are sensitive to as low values of $4\left|U_{e 3}\right|^{2}$ as $10^{-3}-10^{-5}$. This is more than two orders of magnitude better than the current bounds [4] or those expected from the planned long baseline experiments.

\section{F. Level crossing schemes and initial conditions}

In the basis of flavor eigenstates $\left(\nu_{e}, \nu_{\mu}, \nu_{\tau}\right)$, the evolution of neutrinos at densities $\rho<10^{6} \mathrm{~g} / \mathrm{cc}$ relevant for neutrino conversion (see below) is described by a Schrödingerlike equation with the effective Hamiltonian

$$
H=\frac{\mathcal{M}^{2}}{2 E}+\mathcal{V}=\frac{1}{2 E}\left(\begin{array}{lll}
m_{e e}^{2}+2 E V & m_{e \mu}^{2} & m_{e \tau}^{2} \\
m_{e \mu}^{2} & m_{\mu \mu}^{2} & m_{\mu \tau}^{2} \\
m_{e \tau}^{2} & m_{\mu \tau}^{2} & m_{\tau \tau}^{2}
\end{array}\right),
$$

where $\mathcal{V} \approx \operatorname{Diag}(V, 0,0)$, and $V=\sqrt{2} G_{F} n_{e}$ is the effective potential for the electron neutrinos due to their charged current interactions with electrons.

Since any rotation in the $\left(\nu_{\mu}-\nu_{\tau}\right)$ subspace does not affect the physics, it is convenient to perform a rotation of the neutrino states $\left(\nu_{e}, \nu_{\mu}, \nu_{\tau}\right) \rightarrow\left(\nu_{e}, \nu_{\mu^{\prime}}, \nu_{\tau^{\prime}}\right)$, which diagonalizes the $\left(\nu_{\mu}, \nu_{\tau}\right)$ submatrix of Eq. (42) [47]. (The potential $V$ appears only in the element $H_{e e}$, and hence is not affected by this rotation.) The effective Hamiltonian in the new basis becomes

$$
H=\frac{1}{2 E}\left(\begin{array}{lll}
m_{e e}^{2}+2 E V & m_{e \mu^{\prime}}^{2} & m_{e \tau^{\prime}}^{2} \\
m_{e \mu^{\prime}}^{2} & m_{\mu^{\prime} \mu^{\prime}}^{2} & 0 \\
m_{e \tau^{\prime}}^{2} & 0 & m_{\tau^{\prime} \tau^{\prime}}^{2}
\end{array}\right) .
$$


At $V \gg m_{i j}^{2} /(2 E)$, the off-diagonal terms can be neglected and the Hamiltonian (43) becomes diagonal:

$$
H \approx \operatorname{Diag}\left(V, m_{\mu^{\prime} \mu^{\prime}}^{2}, m_{\tau^{\prime} \tau^{\prime}}^{2}\right) .
$$

That is, the basis states $\left(\nu_{e}, \nu_{\mu^{\prime}}, \nu_{\tau^{\prime}}\right)$ are the matter eigenstates. These are the states that arrive at the conversion regions as independent (incoherent) states and transform in this region independently.

Notice that a difference between the potentials of $\nu_{\mu}$ and $\nu_{\tau}$ appears in the second order in the weak interactions due to difference of masses of the $\mu$ and $\tau$ charged leptons [48]:

$$
V_{\mu \tau} \approx V \frac{3 G_{F} m_{\tau}^{2}}{2 \sqrt{2} \pi^{2} Y_{e}}\left(\ln \frac{m_{W}^{2}}{m_{\tau}^{2}}-1+\frac{Y_{n}}{3}\right) \approx 10^{-4} V,
$$

where $m_{\tau}$ is the $\tau$ mass, $m_{W}$ is the $W$-boson mass, and $Y_{e}$ and $Y_{n}$ are the numbers of electrons and the neutrons per nucleon respectively. Therefore a complete form of the matrix of potentials is $\mathcal{V} \approx \operatorname{Diag}\left(V, 0, V_{\mu \tau}\right)$.

The potential $V_{\mu \tau}$ becomes important at high densities. One has

$$
V_{\mu \tau} \sim \Delta m_{\text {atm }}^{2} / 2 E \approx 2 m_{\mu \tau}^{2} / 2 E \text { at } \rho_{\mu \tau} \sim 10^{7}-10^{8} \mathrm{~g} / \mathrm{cc} .
$$

At $\rho \gg \rho_{\mu \tau}$, and in particular in the region of the neutrinosphere, the potentials $V$ and $V_{\mu \tau}$ dominate over the other terms in the Hamiltonian, and the Hamiltonian becomes approximately diagonal: $H \approx \mathcal{V} \approx \operatorname{Diag}\left(V, 0, V_{\mu \tau}\right)$. This means that at high densities the flavor states coincide with the eigenstates in medium.

Let us recall that the non-electron neutrinos are produced in the neutral current processes which are flavor blind; i.e., they produce a coherent mixture of matter eigenstates. This coherence, however, disappears in the evolution that follows. The $\nu_{e}$ departs from the coherent state due to the large potential $V$, whereas the coherence of $\nu_{\mu}$ and $\nu_{\tau}$ is broken by $V_{\mu \tau}$.

In the interval of densities where $V_{\mu \tau} \ll \Delta m_{\text {atm }}^{2} / 2 E \ll V$, the potential $V_{\mu \tau}$ can be neglected, so that we arrive at the Hamiltonian (43) with eigenstates $\left(\nu_{e}, \nu_{\mu^{\prime}}, \nu_{\tau^{\prime}}\right)$. In the region $V_{\mu \tau} \sim \Delta m_{\text {atm }}^{2} / 2 E$, the level crossing occurs in the antineutrino channel [25], which leads to the transitions $\bar{\nu}_{\mu}$ $\rightarrow \bar{\nu}_{\tau}^{\prime}$ and $\bar{\nu}_{\tau} \rightarrow \bar{\nu}_{\mu}^{\prime}$. In the neutrino channel, we have $\nu_{\mu}$ $\rightarrow \nu_{\mu}^{\prime}$ and $\nu_{\tau} \rightarrow \nu_{\tau}^{\prime}$.

Since the initial fluxes of $\stackrel{(-)}{\nu}_{\mu}$ and ${ }^{(-)}{ }_{\tau}$ are equal $\left(F_{x}^{0}\right)$, we get that the fluxes of ${ }^{(-)}{ }_{\mu^{\prime}}$ and ${ }^{(-)}{ }_{\tau^{\prime}}$ will be also equal $\left(F_{x}^{0}\right)$. Therefore $\left(\nu_{e}, \nu_{\mu^{\prime}}, \nu_{\tau^{\prime}}\right)$ with fluxes $\left(F_{e}^{0}, F_{x}^{0}, F_{x}^{0}\right)$ and correspondingly $\left(\bar{\nu}_{e}, \bar{\nu}_{\mu^{\prime}}, \bar{\nu}_{\tau^{\prime}}\right)$ with fluxes $\left(F_{e}^{0}, F_{x}^{0}, F_{x}^{0}\right)$ can be considered as the initial state in our task.

The Hamiltonian (43) allows us easily to construct the level crossing scheme. In Fig. 5, we show the generic level crossing diagrams for the normal and inverted mass hierarchies, for small $\theta_{\odot}$ (SMA) as well as large $\theta_{\odot}$ (LMA or

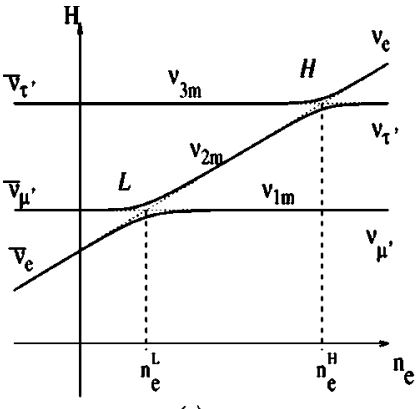

(a)

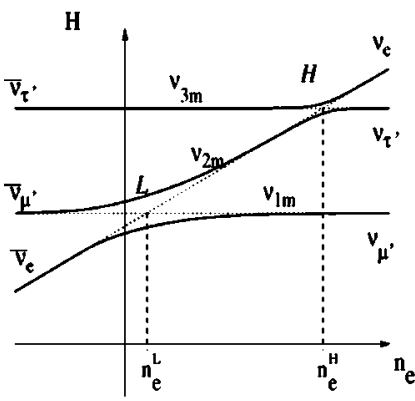

(c)

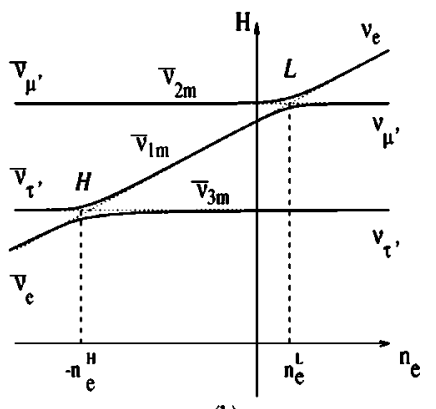

(b)

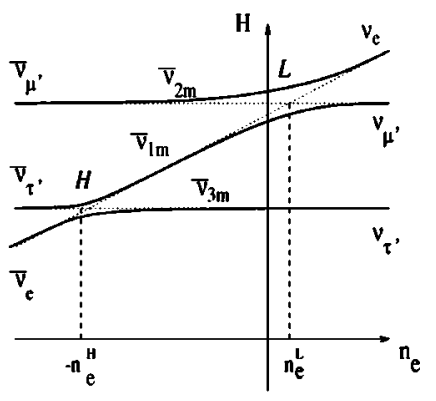

(d)
FIG. 5. The level crossing diagrams for (a) the normal mass hierarchy and small $\theta_{\odot}$, (b) the inverted mass hierarchy and small $\theta_{\odot}$, (c) the normal mass hierarchy and large $\theta_{\odot}$, (d) the inverted mass hierarchy and large $\theta_{\odot}$. Solid lines show the eigenvalues of the effective Hamiltonian as functions of the electron number density. The dashed lines correspond to energies of flavor levels $\nu_{e}$, $\nu_{\mu^{\prime}}$, and $\nu_{\tau^{\prime}}$. The part of the plot with $n_{e}<0$ corresponds to the antineutrino channel.

VO). The diagonal elements of the Hamiltonian (43), $H_{i i}\left(n_{e}\right)\left(i=e, \mu^{\prime}, \tau^{\prime}\right)$, determine the energies of the flavor states shown by the dotted lines. The crossing of these levels indicates a resonance. $H$ and $L$ are the two resonances at higher and lower densities respectively, Eqs. (23) and (24). The solid lines represent the eigenvalues of the Hamiltonian (43).

In the case of antineutrinos, the effective potential $V$ for the $\bar{\nu}_{e}$ has the opposite sign: $V=-\sqrt{2} G_{F} n_{e}$. The antineutrinos can then be represented on the same level crossing diagram, as neutrinos traveling through matter with "effectively" negative $n_{e}$. The half-plane with positive values of $n_{e}$ then describes neutrinos and the half-plane with negative values of $n_{e}$ describes antineutrinos.

The neutrinos (antineutrinos) are produced inside a supernova in regions of high matter density. On their way towards the Earth, they travel through a medium with almost monotonically decreasing density, towards the vacuum where both neutrinos and antineutrinos have vanishing effective potentials. This corresponds to starting at the right (left) extreme ends of the $n_{e}$ axis in Fig. 5, and moving towards $n_{e}=0$.

The $H$ resonance lies in the neutrino channel for the normal hierarchy and in the antineutrino channel for the inverted hierarchy. The $L$ resonance lies in the neutrino channel for both the hierarchies as long as the solar neutrino solution is SMA or LMA. For the VO solution, the $L$ resonance may lie in either of the two channels, neutrinos or antineutrinos. 


\section{CONVERSION PROBABILITIES \\ AND THE NEUTRINO FLUXES AT THE DETECTORS: THE CASE OF NORMAL MASS HIERARCHY}

In this section, we derive general expressions for the transition probabilities using the level crossing scheme for the normal mass hierarchy as shown in Figs. 5a and 5c. (The inverted hierarchy will be discussed separately in Sec. V.)

\section{A. Probabilities of conversion inside the star}

As has been discussed in Sec. II F, the neutrino fluxes arise from the central part of the star, in the region of high density. For $\rho \gg \rho_{H}, \rho_{L}$, where all the mixings are highly suppressed, the flavor states $\left(\nu_{e}, \nu_{\mu^{\prime}}, \nu_{\tau^{\prime}}\right)$ coincide with the eigenstates in the medium:

$$
\nu_{3 m}=\nu_{e}, \quad \nu_{2 m}=\nu_{\tau^{\prime}}, \quad \nu_{1 m}=\nu_{\mu^{\prime}} .
$$

The original fluxes of neutrino eigenstates in the medium equal

$$
F_{1 m}^{0}=F_{x}^{0}, \quad F_{2 m}^{0}=F_{x}^{0}, \quad F_{3 m}^{0}=F_{e}^{0} .
$$

Let us calculate the fluxes of the mass eigenstates $\nu_{i}$ at the surface of the star. These states, being the eigenstates of the Hamiltonian in vacuum, travel independently to the surface of the earth.

Taking into account that the dynamics of transitions in the two resonance layers are independent (see Sec. II D), the fluxes of neutrino mass eigenstates at the surface of the star can be written down directly by tracing the path of the neutrinos in the level crossing diagram. We find the modifications of fluxes in terms of the flip probabilities $P_{H}, P_{L}, \bar{P}_{H}$ and $\bar{P}_{L}$ introduced in Sec. II E.

Let us first calculate the flux of $\nu_{1}$ at the surface of the star. There are three independent contributions to this flux, from the initial $\nu_{e}, \nu_{\mu^{\prime}}$ and $\nu_{\tau^{\prime}}$ fluxes. The probability that the original state $\nu_{e}$ (which coincides with $\nu_{3 m}$ in the production region) arrives at the surface of the star as $\nu_{1}$ is $P_{H} P_{L}$, since the state has to flip to the other matter eigenstate at both the resonances. The contribution to the final $\nu_{1}$ flux from the original $\nu_{e}$ flux is then $P_{H} P_{L} F_{e}^{0}$. Similarly, the contributions from the original $\nu_{\mu^{\prime}}$ and $\nu_{\tau^{\prime}}$ equal (1 $\left.-P_{L}\right) F_{x}^{0}$ and $P_{L}\left(1-P_{H}\right) F_{x}^{0}$ respectively. The total $\nu_{1}$ flux at the surface of the star equals the sum of the three contributions:

$$
F_{1}=P_{H} P_{L} F_{e}^{0}+\left(1-P_{H} P_{L}\right) F_{x}^{0}
$$

Similarly, the fluxes of neutrino mass eigenstates $\nu_{2}$ and $\nu_{3}$ arriving at the surface of the star are

$$
\begin{aligned}
& F_{2}=\left(P_{H}-P_{H} P_{L}\right) F_{e}^{0}+\left(1-P_{H}+P_{H} P_{L}\right) F_{x}^{0}, \\
& F_{3}=\left(1-P_{H}\right) F_{e}^{0}+P_{H} F_{x}^{0} .
\end{aligned}
$$

Because of the divergence of the wave packets, any coherence between the mass eigenstates is lost on the way to the Earth. Indeed, over a distance $L$, the two wave packets corresponding to two mass eigenstates with a given $\Delta m^{2}$ and having an energy $E$ separate from each other by a distance

$$
\Delta L=\frac{\Delta m^{2}}{2 E^{2}} L
$$

Even for the smallest $\Delta m^{2} \sim 10^{-10} \mathrm{eV}^{2}$, for $E \sim 10 \mathrm{MeV}$ and $L \sim 10 \mathrm{kpc} \sim 10^{22} \mathrm{~cm}$, we get $\Delta L \sim 10^{-2} \mathrm{~cm}$. The lengths of the individual wave packets are $\sigma \lesssim 1 / T \sim 10^{-11} \mathrm{~cm}$ (where $T$ is the temperarture of the production region) is much smaller.

The spread of the wave packets implies that the neutrinos arrive at the surface of the earth as incoherent fluxes of the mass eigenstates. Up to a geometrical factor of $1 / L^{2}$, they coincide with the fluxes given in Eqs. (48),(49). We can rewrite them as

$$
F_{i}=a_{i} F_{e}^{0}+\left(1-a_{i}\right) F_{x}^{0}
$$

with

$$
a_{1}=P_{H} P_{L}, \quad a_{2}=P_{H}\left(1-P_{L}\right), \quad a_{3}=1-P_{H} .
$$

The factor of $1 / L^{2}$ is implicit in the fluxes at the Earth.

Using Eq. (51), we find the net flux of electron neutrinos at the Earth:

$$
F_{e}=\sum_{i}\left|U_{e i}\right|^{2} F_{i}=F_{e}^{0} \sum_{i}\left|U_{e i}\right|^{2} a_{i}+F_{x}^{0}\left(1-\sum_{i}\left|U_{e i}\right|^{2} a_{i}\right),
$$

where we have taken into account the unitarity condition $\Sigma\left|U_{e i}\right|^{2}=1$. The final electron neutrino flux reaching the Earth can thus be written as

$$
F_{e}=p F_{e}^{0}+(1-p) F_{x}^{0},
$$

where

$$
\begin{aligned}
p & \equiv \sum_{i}\left|U_{e i}\right|^{2} a_{i} \\
& =\left|U_{e 1}\right|^{2} P_{H} P_{L}+\left|U_{e 2}\right|^{2}\left(P_{H}-P_{H} P_{L}\right)+\left|U_{e 3}\right|^{2}\left(1-P_{H}\right)
\end{aligned}
$$

According to Eq. (54), $p$ may be interpreted as the total survival probability of electron neutrinos.

The original total flux of the neutrinos $\nu_{e}, \nu_{\mu}, \nu_{\tau}$ is $F_{e}^{0}$ $+2 F_{x}^{0}$. Using the conservation of flux, we find the combined flux of $\nu_{\mu}$ and $\nu_{\tau}$ at the Earth $\left(F_{\mu}+F_{\tau}\right)$ as

$$
F_{\mu}+F_{\tau}=(1-p) F_{e}^{0}+(1+p) F_{x}^{0} .
$$

Note that the final fluxes of the flavor states at the Earth, Eqs. (54),(56), can be written in terms of only the survival probability $p$. This is a consequence of two facts: (1) at each transition, one of the neutrinos is decoupled so that the task reduces to $2 \nu$ mixing, and (2) the original fluxes of $\nu_{\mu^{\prime}}$ and $\nu_{\tau^{\prime}}$ are equal. 


\section{B. Conversion probabilities for antineutrinos}

Let us consider the antineutrino transitions. In the high matter density region $\left(\rho \gg \rho_{H}, \rho_{L}\right)$, the antineutrino flavor eigenstates coincide with the eigenstates in the medium as (see Figs. 5a, 5c):

$$
\bar{\nu}_{1 m}=\bar{\nu}_{e}, \quad \bar{\nu}_{2 m}=\bar{\nu}_{\mu^{\prime}}, \quad \bar{\nu}_{3 m}=\bar{\nu}_{\tau^{\prime}}
$$

so that the original fluxes of antineutrino eigenstates in the medium equal

$$
\bar{F}_{1 m}^{0}=F_{e}^{0}, \quad \bar{F}_{2 m}^{0}=F_{x}^{0}, \quad \bar{F}_{3 m}^{0}=F_{x}^{0} .
$$

The small mixing angle $\theta_{e 3}$ is further suppressed in the medium, so the $\bar{\nu}_{e} \leftrightarrow \bar{\nu}_{3}$ transitions are negligible. The state $\bar{\nu}_{3 m}$, being far from the level crossings, propagates adiabatically: $\bar{\nu}_{\tau^{\prime}} \rightarrow \bar{\nu}_{3}$. Depending on the parameters of the solution for the solar neutrino problem, the propagation of the other two states may be adiabatic or non-adiabatic [19]. From considerations similar to those in the neutrino channel, we get

$$
F_{\bar{e}}=\bar{p} F_{\bar{e}}^{0}+(1-\bar{p}) F_{x}^{0},
$$

where $\bar{p}$, the survival probability of $\bar{\nu}_{e}$, equals

$$
\bar{p}=\left|U_{e 1}\right|^{2}\left(1-\bar{P}_{L}\right)+\left|U_{e 2}\right|^{2} \bar{P}_{L}
$$

In the case of completely adiabatic propagation, $\bar{P}_{L}=0$ and

$$
\bar{p}=\left|U_{e 1}\right|^{2}
$$

Let $F_{\mu}+F_{\tau}^{-}$be the combined flux of $\bar{\nu}_{\mu}$ and $\bar{\nu}_{\tau}$. From Eq. (59) and the conservation of flux, we get the combined flux of non-electron antineutrinos:

$$
F_{\bar{\mu}}+F_{\bar{\tau}}^{-}=(1-\bar{p}) F_{\bar{e}}^{0}+(1+\bar{p}) F_{x}^{0} .
$$

From Eqs. (56) and (62), the total flux of the non-electron neutrinos (including antineutrinos) is

$$
\begin{aligned}
4 F_{x} & =F_{\mu}+F_{\tau}+F_{\bar{\mu}}+F_{\bar{\tau}} \\
& =(1-p) F_{e}^{0}+(2+p+\bar{p}) F_{x}^{0}+(1-\bar{p}) F_{\bar{e}}^{0} .
\end{aligned}
$$

Summarizing, Eqs. (54),(59),(63) can be written in the compact notation

$$
\left(\begin{array}{c}
F_{e} \\
F_{\bar{e}} \\
4 F_{x}
\end{array}\right)=\left(\begin{array}{ccc}
p & 0 & 1-p \\
0 & \bar{p} & 1-\bar{p} \\
1-p & 1-\bar{p} & 2+p+\bar{p}
\end{array}\right)\left(\begin{array}{c}
F_{e}^{0} \\
F_{\bar{e}}^{0} \\
F_{x}^{0}
\end{array}\right) .
$$

The above general expression holds for both the normal and inverted mass hierarchies. The survival probabilities $p$ and $\bar{p}$ for the normal hierarchy are given in Eqs. (55) and (60) respectively. These probabilities depend on the specific parameters of the masses and mixing scheme.

\section{Earth matter effects on the $\nu_{e}$ spectrum}

The neutrino trajectory inside the Earth before reaching the detector depends on the direction of the supernova relative to the Earth and the time of the day. The comparison of signals from different detectors would allow one to reveal the Earth matter effects. Also certain features of the energy spectra can reveal the Earth matter effect even from the observations in one detector.

The mass eigenstates arriving at the surface of the Earth oscillate in the Earth matter. Let $P_{i e}$ be the probability that a mass eigenstate $\nu_{i}$ entering the earth reaches the detector as a $\nu_{e}$. The flux of $\nu_{e}$ at the detector is

$$
F_{e}^{D}=\sum_{i} P_{i e} F_{i}
$$

Inserting $F_{i}$ from Eq. (51), we get

$$
F_{e}^{D}=F_{e}^{0} \sum a_{i} P_{i e}+F_{x}^{0}\left(1-\sum_{i} a_{i} P_{i e}\right),
$$

where $a_{i}$ 's are as defined in Eq. (52), and we have used the unitarity condition $\Sigma_{i} P_{i e}=1$. Thus, the $\nu_{e}$ flux at the detector can be written as

$$
F_{e}^{D}=p^{D} F_{e}^{0}+\left(1-p^{D}\right) F_{x}^{0},
$$

with

$$
p^{D}=\sum_{i} a_{i} P_{i e}
$$

Comparing $F_{e}^{D}$, Eq. (67), with $F_{e}$, Eq. (54), we find that the difference in the $\nu_{e}$ fluxes at the detector due to the propagation in the Earth equals

$$
F_{e}^{D}-F_{e}=\left(p^{D}-p\right)\left(F_{e}^{0}-F_{x}^{0}\right)
$$

where $p$ is given in Eq. (55). The Earth matter effect can be quantified by the difference of probabililty $\left(p^{D}-p\right)$ :

$$
p^{D}-p=\sum_{i} a_{i}\left(P_{i e}-\left|U_{e i}\right|^{2}\right)
$$

Using definitions in Eqs. (52) and $\Sigma_{i} P_{i e}=1$, we can write, explicitly,

$$
\begin{aligned}
p^{D}-p= & P_{H}\left(P_{2 e}-\left|U_{e 2}\right|^{2}\right)\left(1-2 P_{L}\right) \\
& +\left(P_{3 e}-\left|U_{e 3}\right|^{2}\right)\left(1-P_{H}-P_{H} P_{L}\right) .
\end{aligned}
$$

The second term in Eq. (71) can be neglected. Indeed, inside the Earth, $\nu_{3}$ oscillates with a very small depth:

$$
P_{3 e}-\left|U_{e 3}\right|^{2} \lesssim\left(\frac{2 E V_{\text {Earth }}}{\Delta m_{\text {atm }}^{2}}\right) \sin ^{2} 2 \theta_{e 3},
$$

where $V_{\text {Earth }}$ is the effective potential of $\nu_{e}$ in the Earth. For neutrino energies of $5-50 \mathrm{MeV}$, we have $2 E V_{E a r t h} / \Delta m_{\text {atm }}^{2}$ $\lesssim 10^{-2}$. Moreover, $\sin ^{2} 2 \theta_{e 3} \leqslant 0.1$, so that 


$$
P_{3 e}-\left|U_{e 3}\right|^{2} \leqslant 10^{-3} \text {. }
$$

Finally, we can write

$$
p^{D}-p \approx P_{H}\left(P_{2 e}-\left|U_{e 2}\right|^{2}\right)\left(1-2 P_{L}\right) .
$$

In general, when the signals from two detectors $D 1$ and $D 2$ are compared, we get the difference of fluxes

$$
F_{e}^{D 1}-F_{e}^{D 2} \approx P_{H}\left(1-2 P_{L}\right)\left(P_{2 e}^{(1)}-P_{2 e}^{(2)}\right)\left(F_{e}^{0}-F_{x}^{0}\right),
$$

where $P_{2 e}^{(1)}$ and $P_{2 e}^{(2)}$ are the $\nu_{e} \leftrightarrow \nu_{2}$ oscillation probabilities for the detectors $D 1$ and $D 2$ correspondingly.

According to Eq. (75) the Earth matter effect is factorized: it is proportional to the difference in the original $\nu_{e}$ and $\nu_{x}$ fluxes, the conversion factor $P_{H}\left(1-2 P_{L}\right)$ inside the star, and the difference of Earth oscillation probabilities $P_{2 e}$ at the two detectors. Let us consider these factors separately.

(1) $F_{e}^{0}-F_{x}^{0}$ : Since the $\nu_{e}$ spectrum is softer than the $\nu_{x}$ spectrum, and the luminosities of both the spectra are similar in magnitude [49], the term $\left(F_{e}^{0}-F_{x}^{0}\right)$ is positive at low energies and becomes negative at higher energies where the $\nu_{x}$ flux overwhelms the $\nu_{e}$ flux. Therefore, the Earth effect has a different sign for low and high energies, and there exists a critical energy $E_{c}$ such that $F_{e}^{0}\left(E_{c}\right)=F_{x}^{0}\left(E_{c}\right)$, where this change of sign takes place. Since the cross section of the neutrino interactions increases with energy, the Earth effect is expected to be more significant at higher energies (if all the other factors are only weakly sensitive to the neutrino energy).

(2) $P_{H}\left(1-2 P_{L}\right)$ : This factor characterizes the neutrino conversions inside the star. $P_{H}$ can be looked upon as a suppression factor due to the conversions at the higher resonance. Indeed, in the limit of $P_{H} \rightarrow 1$, Eq. (74) reduces to the expression for the earth effects in the case of two neutrino mixing:

$$
\left[p^{D}-p\right]_{2 \nu}=\left(P_{2 e}-\left|U_{e 2}\right|^{2}\right)\left(1-2 P_{L}\right)
$$

which is equivalent to the one used in literature [50] in the context of day-night effect for solar neutrinos. Therefore Eq. (74) can be looked upon as the Earth matter effect due to the two neutrino mixing (76) suppressed by a factor of $P_{H}$. The mixing of the third neutrino thus plays a major role, making the expected Earth effects in the case of supernova neutrinos smaller than those expected in the case of solar neutrinos for the same mixing scheme and in the same energy range. If the $H$ resonance is completely adiabatic, the Earth effect vanishes: all the $\nu_{e}$ produced are converted to $\nu_{3}$ in the star, and the Earth matter effect on $\nu_{3}$ is negligibly small [as we have established through Eq. (72)].

(3) $P_{2 e}^{(1)}-P_{2 e}^{(2)}$ : If the neutrino trajectory crosses only the mantle of the Earth, one can use a constant density approximation which gives

$$
\begin{aligned}
P_{2 e}^{(1)}-P_{2 e}^{(2)} \approx & \sin 2 \theta_{e 2}^{m} \sin \left(2 \theta_{e 2}^{m}-2 \theta_{e 2}\right) \\
& \times\left[\sin ^{2}\left(\frac{\pi d_{1}}{l_{m}}\right)-\sin ^{2}\left(\frac{\pi d_{2}}{l_{m}}\right)\right] .
\end{aligned}
$$

Here $\theta_{e 2}^{m}$ and $l_{m}$ are the mixing angle and oscillation length inside the Earth respectively, and $d_{i}$ is the distance traveled by the neutrinos inside the Earth before reaching the detector $D_{i}$. The first two terms on the right hand side of Eq. (77) are positive for the scenarios with the SMA and LMA solutions, so that the sign of $\left(P_{2 e}^{(1)}-P_{2 e}^{(2)}\right)$ is the same as the sign of the term inside the square brackets in Eq. (77). For the scenario with the VO solution, the Earth matter effects are negligible: for $\Delta m^{2} \sim 10^{-10} \mathrm{eV}^{2}$, the mixing in the Earth matter is highly suppressed, so that $\sin 2 \theta_{e 2}^{m}$ in Eq. (77) is very small.

If neutrinos cross both the mantle and the core, the parametric enhancement of oscillations may occur, which leads to the appearance of parametric peaks apart from the peaks due to the MSW resonances in the core and the mantle [51]. Correspondingly the factor $\left(P_{2 e}^{(1)}-P_{2 e}^{(2)}\right)$ will be a more complicated function of the neutrino energy.

To summarize, the Earth matter effects on the $\nu_{e}$ spectrum can be significant only for the scenarios with the SMA or LMA or LOW solutions. Moreover, the $H$ resonance needs to be non-adiabatic in the case of normal mass hierarchy.

\section{Earth matter effects on the $\bar{\nu}_{e}$ spectrum}

The oscillation effects are determined by the survival probability of the electron antineutrinos, $\bar{p}^{D}$, at the detector. Considerations similar to those for Eq. (60) lead to

$$
\bar{p}^{D}=\bar{P}_{1 e}\left(1-\bar{P}_{L}\right)+\bar{P}_{2 e} \bar{P}_{L} .
$$

Then from Eqs. (59), (60) and (78), we obtain

$$
F_{\bar{e}}^{D}-F_{e}^{-}=\left(\bar{P}_{1 e}-\left|U_{e 1}\right|^{2}\right)\left(1-2 \bar{P}_{L}\right)\left(F_{e}^{0}-F_{x}^{0}\right),
$$

where we have neglected the oscillations of $\bar{\nu}_{3}$ inside the Earth. Generalizing the result in Eq. (79), we find the difference in the fluxes at two detectors $D 1$ and $D 2$ :

$$
F_{\bar{e}}^{D 1}-F_{\bar{e}}^{D 2} \approx\left(\bar{P}_{1 e}^{(1)}-\bar{P}_{1 e}^{(2)}\right)\left(1-2 \bar{P}_{L}\right)\left(F_{\bar{e}}^{0}-F_{x}^{0}\right) .
$$

The Earth matter effects for the antineutrinos are thus also factorized: they are proportional to the difference in the original $\nu_{e}$ and $\nu_{x}$ fluxes, the factor of $\left(1-2 \bar{P}_{L}\right)$ which depends on the conversions inside the star, and the difference $\left(\bar{P}_{1 e}^{(1)}-\bar{P}_{1 e}^{(2)}\right)$ between the oscillation probabilities inside the Earth for the neutrinos reaching the two detectors. Note that due to the absence of the $H$ resonance in the antineutrino channel, there is no suppression factor similar to $P_{H}$, Eq. (75), in the neutrino case.

The factor $\left(F_{\bar{e}}^{0}-F_{x}^{0}\right)$ is positive at low energies and negative at high energies. The matter effects then change sign at an energy $\bar{E}_{c}$ where $F_{e}^{0}\left(\bar{E}_{c}\right)=F_{x}^{0}\left(\bar{E}_{c}\right)$. Since the neutrino cross section increases with energy, the observed effect is expected to be larger at higher energies (if all the other factors are only weakly sensitive to the antineutrino energy).

In the approximation of a constant density, the factor depending on the oscillation probabilities inside the Earth is 


$$
\begin{aligned}
\bar{P}_{1 e}^{(1)}-\bar{P}_{1 e}^{(2)} \approx & -\sin 2 \bar{\theta}_{e 2}^{m} \sin \left(2 \bar{\theta}_{e 2}^{m}-2 \theta_{e 2}\right) \\
& \times\left[\sin ^{2}\left(\frac{\pi d_{1}}{l_{m}}\right)-\sin ^{2}\left(\frac{\pi d_{2}}{l_{m}}\right)\right],
\end{aligned}
$$

where $\bar{\theta}_{e 2}^{m}$ is the mixing angle inside the Earth for the antineutrinos. For the antineutrino channel $\bar{\theta}_{e 2}^{m}<\theta_{e 2} \ll 1$ for the SMA solution and $\bar{\theta}_{e 2}^{m}$ is strongly suppressed by matter in the VO case. Therefore the Earth matter effects on the $\bar{\nu}_{e}$ spectrum can be significant only for the scenario with the LMA (as well as LOW) solution. In this scenario, $\sin 2 \bar{\theta}_{e 2}^{m}>0$ and $\sin \left(2 \bar{\theta}_{e 2}^{m}-2 \theta_{e 2}\right)<0$, so that according to Eq. (81), the sign of $\left(\bar{P}_{1 e}^{(1)}-\bar{P}_{1 e}^{(2)}\right)$ is the same as the sign of the oscillation term inside the square brackets in Eq. (81).

To summarize, in the case of normal mass hierarchy, the Earth matter effects on the $\bar{\nu}_{e}$ spectrum are significant only for the scenario with the LMA solution. The effects are practically unaffected by the mixing of $\bar{\nu}_{3}$ and change sign at an energy $\bar{E}_{c}$ such that $F_{\bar{e}}^{0}\left(\bar{E}_{c}\right)=F_{x}^{0}\left(\bar{E}_{c}\right)$.

\section{EFFECTS OF NEUTRINO CONVERSION FOR THE MASS SPECTRA WITH NORMAL HIERARCHY}

In this section, we shall consider the neutrino conversion effects for specific $3 \nu$ schemes with the normal mass hierarchy. The general expressions for the transition probabilities and the neutrino fluxes at the detectors are given in Sec. III, where they are expressed in terms of the total survival probabilities $p$ and $\bar{p}$ of electron neutrinos and electron antineutrinos respectively. The values of $p$ and $\bar{p}$ need to be calculated separately for each specific scheme. Notice that, given a scheme, the least known parameter is $\left|U_{e 3}\right|^{2}$. We shall consider the effects of neutrino conversion in the three possible regions of $\left|U_{e 3}\right|^{2}$ : these correspond to regions I, II and III as described in Sec. II (see also Figs. 2 and 3.)

In Table I, we tabulate the values of $p$ and $\bar{p}$ for all the cases under discussion. In Table II, we summarize the information regarding (i) the channels in which the neutronization peak appears: " $\approx \nu_{x}$ " means an almost complete disappearance of the peak from the $\nu_{e}$ channel-this corresponds to a suppression by a factor of $\left|U_{e 3}\right|^{2} \leqslant 0.03$, (ii) the qualitative nature of the final $\nu_{e}$ and $\bar{\nu}_{e}$ spectra: "soft" implies the original $\nu_{e}$ or $\bar{\nu}_{e}$ spectrum, "hard" denotes the original $\nu_{x}$ spectrum, and "composite" signifies a mixture of both the soft and hard components, (iii) the Earth matter effects: $\sqrt{ }$ denotes the possibility of significant Earth effects, " $\approx 0$ ", implies that Earth matter effects are practically absent.

\section{A. Scheme with the SMA solution}

The mass and flavor spectrum of the scheme is shown in Fig. 6. The non-electron neutrinos $\nu_{\mu}$ and $\nu_{\tau}$ mix strongly in the mass eigenstates $\nu_{2}$ and $\nu_{3}$. The electron flavor is weakly mixed: it is mainly in $\nu_{1}$ with small admixtures in the heavy states. The solar neutrino data are explained via the small mixing angle MSW solution, with the parameters as given in
TABLE I. The values of the survival probabilities $p$ and $\bar{p}$ for various scenarios. $[x, y]$ indicates that the value of the survival

\begin{tabular}{|c|c|c|c|c|}
\hline & & & $p$ & $\bar{p}$ \\
\hline \multirow[t]{6}{*}{ I } & SMA & Normal & $\left|U_{e 3}\right|^{2}$ & 1 \\
\hline & & Inverted & $P_{L}$ & $\left|U_{e 3}\right|^{2}$ \\
\hline & LMA & Normal & $\left|U_{e 3}\right|^{2}$ & $\cos ^{2} \theta_{\odot}$ \\
\hline & & Inverted & $\sin ^{2} \theta_{\odot}$ & $\left|U_{e 3}\right|^{2}$ \\
\hline & $\mathrm{VO}$ & Normal & $\left|U_{e 3}\right|^{2}$ & {$\left[\sin ^{2} \theta_{\odot}, \cos ^{2} \theta_{\odot}\right]$} \\
\hline & & Inverted & {$\left[\sin ^{2} \theta_{\odot}, \cos ^{2} \theta_{\odot}\right]$} & $\left|U_{e 3}\right|^{2}$ \\
\hline \multirow[t]{6}{*}{ II } & SMA & Normal & {$\left[\left|U_{e 3}\right|^{2}, P_{L}\right]$} & 1 \\
\hline & & Inverted & $P_{L}$ & $\bar{P}_{H}$ \\
\hline & LMA & Normal & $\sin ^{2} \theta_{\odot} P_{H}$ & $\cos ^{2} \theta_{\odot}$ \\
\hline & & Inverted & $\sin ^{2} \theta_{\odot}$ & $\cos ^{2} \theta_{\odot} \bar{P}_{H}$ \\
\hline & VO & Normal & {$\left[\left|U_{e 3}\right|^{2}, \cos ^{2} \theta_{\odot}\right]$} & {$\left[\sin ^{2} \theta_{\odot}, \cos ^{2} \theta_{\odot}\right]$} \\
\hline & & Inverted & {$\left[\sin ^{2} \theta_{\odot}, \cos ^{2} \theta_{\odot}\right]$} & {$\left[\sin ^{2} \theta_{\odot} \bar{P}_{H}, \cos ^{2} \theta_{\odot} \bar{P}_{H}\right]$} \\
\hline \multirow[t]{6}{*}{ III } & SMA & Normal & $P_{L}$ & 1 \\
\hline & & Inverted & $P_{L}$ & 1 \\
\hline & LMA & Normal & $\sin ^{2} \theta_{\odot}$ & $\cos ^{2} \theta_{\odot}$ \\
\hline & & Inverted & $\sin ^{2} \theta_{\odot}$ & $\cos ^{2} \theta_{\odot}$ \\
\hline & $\mathrm{VO}$ & Normal & {$\left[\sin ^{2} \theta_{\odot}, \cos ^{2} \theta_{\odot}\right]$} & {$\left[\sin ^{2} \theta_{\odot}, \cos ^{2} \theta_{\odot}\right]$} \\
\hline & & Inverted & {$\left[\sin ^{2} \theta_{\odot}, \cos ^{2} \theta_{\odot}\right]$} & {$\left[\sin ^{2} \theta_{\odot}, \cos ^{2} \theta_{\odot}\right]$} \\
\hline
\end{tabular}
probability lies between $x$ and $y$.

Eq. (3). The level crossing scheme is shown in Fig. 5a. Both the resonances are in the neutrino channel.

In this scheme,

$$
\left|U_{e 1}\right|^{2} \approx 1, \quad\left|U_{e 2}\right|^{2} \approx \frac{1}{4} \sin ^{2} 2 \theta_{\odot} \sim 10^{-3} .
$$

Let us first consider the antineutrino channels. There is no resonance here, and the mixing in matter is suppressed. As a consequence, the adiabaticity condition is fulfilled in the $L$ layer and $\bar{P}_{L} \approx 0$. Then according to the level crossing scheme (Fig. 5a), the following transitions occur inside the star:

$$
\bar{\nu}_{e} \rightarrow \bar{\nu}_{1}, \quad \bar{\nu}_{\mu^{\prime}} \rightarrow \bar{\nu}_{2}, \quad \bar{\nu}_{\tau^{\prime}} \rightarrow \bar{\nu}_{3}
$$

Using $\left|U_{e 2}\right|^{2} \ll 1$ and $\bar{P}_{L} \approx 0$, we get from Eq. (60) the survival probability for $\bar{\nu}_{e}$ :

$$
\bar{p} \approx\left|U_{e 1}\right|^{2}\left(1-\bar{P}_{L}\right) \approx 1
$$

Let us now consider the neutrino channels. The value of the $\nu_{e}$ survival probability $p$ depends on the region in which the oscillation parameters of the $H$ resonance lie.

Region $I$. Since the $H$ resonance is adiabatic, the level crossing scheme (Fig. 5a) leads to the following transitions:

$$
\nu_{e} \rightarrow \nu_{3}, \quad \nu_{\mu^{\prime}} \rightarrow \nu_{1}, \nu_{2}, \quad \nu_{\tau^{\prime}} \rightarrow \nu_{1}, \nu_{2}
$$

At $P_{H} \approx 0$, the Eq. (55) gives

$$
p \approx\left|U_{e 3}\right|^{2} \leqslant 0.03 \text {. }
$$


TABLE II. The observable effects of various scenarios for (i) the neutronization peak, (ii) the nature of final $\nu_{e}$ and $\bar{\nu}_{e}$ spectra, and (iii) the Earth matter effects. The interpretation of the entries in the table is given in the beginning of Sec. IV.

\begin{tabular}{|c|c|c|c|c|c|c|c|}
\hline & & & \multirow{2}{*}{$\begin{array}{l}\text { Neutronization } \\
\text { peak }\end{array}$} & \multicolumn{2}{|c|}{ Spectrum } & \multicolumn{2}{|c|}{ Earth effects } \\
\hline & & & & $\nu_{e}$ & $\bar{\nu}_{e}$ & $\nu_{e}$ & $\bar{\nu}_{e}$ \\
\hline \multirow[t]{6}{*}{ I } & \multirow[t]{2}{*}{ SMA } & Normal & $\approx \nu_{x}$ & Hard & Soft & $\approx 0$ & $\approx 0$ \\
\hline & & Inverted & $\nu_{e}, \nu_{x}$ & Composite & Hard & $\checkmark$ & $\approx 0$ \\
\hline & \multirow[t]{2}{*}{ LMA } & Normal & $\approx \nu_{x}$ & Hard & Composite & $\approx 0$ & $\checkmark$ \\
\hline & & Inverted & $\nu_{e}, \nu_{x}$ & Composite & Hard & $\checkmark$ & $\approx 0$ \\
\hline & \multirow[t]{2}{*}{ VO } & Normal & $\approx \nu_{x}$ & Hard & Composite & $\approx 0$ & $\approx 0$ \\
\hline & & Inverted & $\nu_{e}, \nu_{x}$ & Composite & Hard & $\approx 0$ & $\approx 0$ \\
\hline \multirow[t]{6}{*}{ II } & \multirow[t]{2}{*}{ SMA } & Normal & $\nu_{e}, \nu_{x}$ & Composite & Soft & $\sqrt{ }$ & $\approx 0$ \\
\hline & & Inverted & $\nu_{e}, \nu_{x}$ & Composite & Composite & $\checkmark$ & $\approx 0$ \\
\hline & \multirow[t]{2}{*}{ LMA } & Normal & $\nu_{e}, \nu_{x}$ & Composite & Composite & $\checkmark$ & $\checkmark$ \\
\hline & & Inverted & $\nu_{e}, \nu_{x}$ & Composite & Composite & $\checkmark$ & $\sqrt{ }$ \\
\hline & \multirow[t]{2}{*}{ VO } & Normal & $\nu_{e}, \nu_{x}$ & Composite & Composite & $\approx 0$ & $\approx 0$ \\
\hline & & Inverted & $\nu_{e}, \nu_{x}$ & Composite & Composite & $\approx 0$ & $\approx 0$ \\
\hline \multirow[t]{6}{*}{ III } & \multirow[t]{2}{*}{ SMA } & Normal & $\nu_{e}, \nu_{x}$ & Composite & Soft & $\checkmark$ & $\approx 0$ \\
\hline & & Inverted & $\nu_{e}, \nu_{x}$ & Composite & Soft & $\checkmark$ & $\approx 0$ \\
\hline & \multirow[t]{2}{*}{ LMA } & Normal & $\nu_{e}, \nu_{x}$ & Composite & Composite & $\sqrt{ }$ & $\checkmark$ \\
\hline & & Inverted & $\nu_{e}, \nu_{x}$ & Composite & Composite & $\sqrt{ }$ & $\sqrt{ }$ \\
\hline & \multirow[t]{2}{*}{ VO } & Normal & $\nu_{e}, \nu_{x}$ & Composite & Composite & $\approx 0$ & $\approx 0$ \\
\hline & & Inverted & $\nu_{e}, \nu_{x}$ & Composite & Composite & $\approx 0$ & $\approx 0$ \\
\hline
\end{tabular}

The flavor transitions are then complete, and independent of the adiabaticity at the $L$ resonance.

The final neutrino (and antineutrino) fluxes are

$$
F_{e} \approx F_{x}^{0}, \quad F_{e}^{-} \approx F_{e}^{0}, \quad 4 F_{x} \approx F_{e}^{0}+3 F_{x}^{0}
$$

Note that $\nu_{e}$ has the hard spectrum $F_{x}^{0}$, while the $\bar{\nu}_{e}$ spectrum is unchanged, therefore $\left.\left\langle E_{\nu_{e}}\right\rangle\right\rangle\left\langle E_{\bar{\nu}_{e}}\right\rangle$, which is a clear signal of mixing.

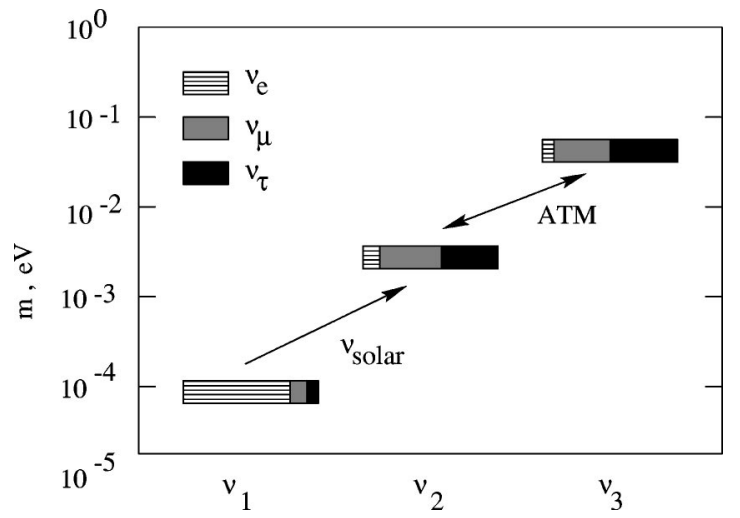

FIG. 6. Neutrino mass and mixing pattern for the scheme with the small mixing angle MSW solution of the solar neutrino problem. The boxes correspond to the mass eigenstates. The sizes of different regions in the boxes show admixtures of different flavors. Weakly hatched regions correspond to the electron flavor, strongly hatched regions depict the muon flavor, black regions represent the tau flavor.
The Earth matter effects on the $\nu_{e}$ spectrum (75) are suppressed by the factor of $P_{H} \approx 0$. Also, since the mixing in the antineutrino channel is suppressed in matter, the Earth matter effects on the $\bar{\nu}_{e}$ spectrum are negligible. As a result, one expects practically the same neutrino (and antineutrino) signal in all detectors.

Region II. Neutrinos jump partially between the third and the second levels in $H$ region, so that the following transitions occur:

$$
\nu_{e} \rightarrow \nu_{1}, \nu_{2}, \nu_{3}, \quad \nu_{\mu^{\prime}} \rightarrow \nu_{1}, \nu_{2}, \quad \nu_{\tau^{\prime}} \rightarrow \nu_{1}, \nu_{2}, \nu_{3}
$$

The $\nu_{e}$ survival probability $p$ is given by the general formula (55). The value of $P_{H}$, and hence the survival probability $p$, depends on the neutrino energy, although this dependence is relatively weak, as demonstrated in Figs. 2 and 3. The $\bar{\nu}_{e}$ spectrum is unchanged while the final $\nu_{e}$ and $\nu_{x}$ spectra are composite.

The Earth matter effects can be observed in the $\nu_{e}$ spectrum, their magnitude being proportional to $P_{H}$, Eq. (75). For $\bar{\nu}_{e}$ spectrum the effect is negligible.

Region III. No conversion occurs at the $H$ resonance, so that in the star

$$
\nu_{e} \rightarrow \nu_{1}, \nu_{2}, \quad \nu_{\mu^{\prime}} \rightarrow \nu_{1}, \nu_{2}, \quad \nu_{\tau^{\prime}} \rightarrow \nu_{3}
$$

From Eqs. (55) and (82) we get, using $P_{H} \approx 1$,

$$
p \approx\left|U_{e 1}\right|^{2} P_{L}+\left|U_{e 2}\right|^{2}\left(1-P_{L}\right) \approx P_{L} .
$$

So we have an unchanged $\bar{\nu}_{e}$ spectrum and composite $\nu_{e}$ and $\nu_{x}$ spectra. 


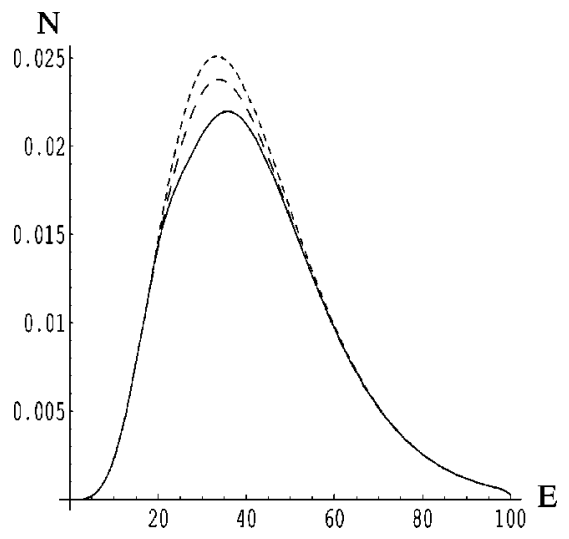

FIG. 7. The Earth matter effects on the $\nu_{e}$ spectrum for $P_{H}$ $=1$ and $P_{L}=0$ in the scheme with the SMA solution $\left(\Delta m^{2}\right.$ $=10^{-5} \mathrm{eV}^{2}, \sin ^{2} 2 \theta_{\odot}=10^{-2}$ ). The dotted, dashed and solid lines show the number of $\nu_{e}-N$ charged current events when the distance traveled by the neutrinos through the mantle of the Earth is $d=0 \mathrm{~km}, d=6000 \mathrm{~km}$, and $d=10000 \mathrm{~km}$ respectively.

The Earth matter effects on the $\nu_{e}$ spectrum in the SMA scheme can be significant in regions II and III, where the factor of $P_{H}$ is of the order of unity. Indeed, in Eq. (77), the value of $\left(P_{2 e}-\left|U_{e 2}\right|^{2}\right)$ can be as large as 0.25 in the energy range of $20-40 \mathrm{MeV}$, where the term $\left(F_{e}^{0}-F_{x}^{0}\right)$ is also significant.

In Fig. 7, we show the $\nu_{e}$ spectrum for different distances travelled by the neutrinos through the Earth. As follows from Fig. 7, the net effect is $\leqslant 10 \%$ even with optimistic values of $P_{H}, P_{L}$ and $\theta_{e 2}$.

\section{B. Scheme with the LMA solution}

The mass and flavor spectrum of the scheme is shown in Fig. 8. The solar neutrino data are explained via the $\nu_{e}$ $\rightarrow \nu_{2}$ resonant conversion inside the Sun with a large vacuum mixing angle (4). The level crossing scheme is shown in Fig. 5c. It differs from the previous one by the mixing at the $L$ resonance. Since the vacuum mixing angle $\theta_{\odot}$ is large, there

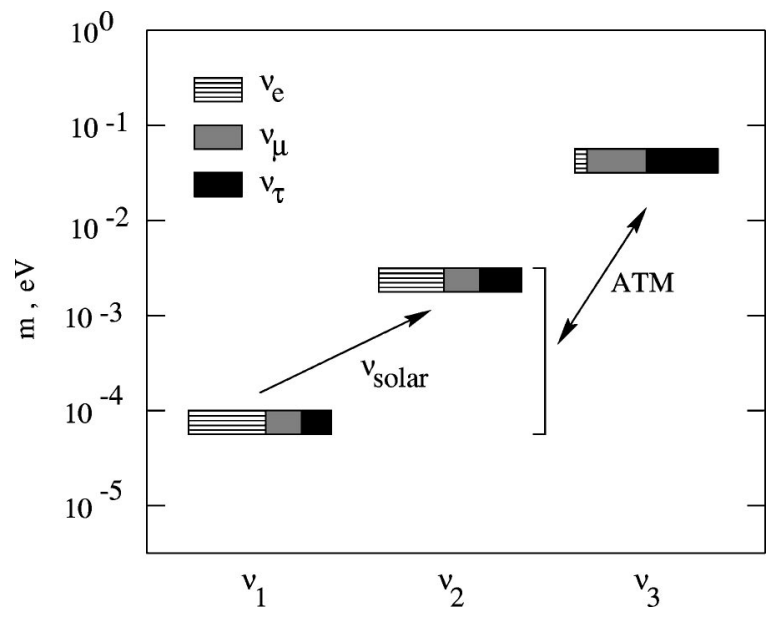

FIG. 8. Neutrino mass and mixing pattern of the scheme with the large mixing angle MSW solution for the solar neutrino problem. is a significant $\bar{\nu}_{e} \leftrightarrow \bar{\nu}_{2}$ conversion even though the antineutrinos do not encounter any resonances. The evolution is adiabatic in the antineutrino channel in both the $H$ and $L$ layers. The following transitions occur:

$$
\bar{\nu}_{e} \rightarrow \bar{\nu}_{1}, \quad \bar{\nu}_{\mu^{\prime}} \rightarrow \bar{\nu}_{2}, \quad \bar{\nu}_{\tau^{\prime}} \rightarrow \bar{\nu}_{3}
$$

Using $\bar{P}_{L} \approx 0$, the $\bar{\nu}_{e}$ survival probability (60) equals

$$
\bar{p} \approx\left|U_{e 1}\right|^{2} \approx \cos ^{2} \theta_{\odot} .
$$

Let us consider transitions in the neutrino channels. The $L$ resonance is adiabatic (Fig. 4), $P_{L} \approx 0$, and from Eq. (55), we get the $\nu_{e}$-survival probability

$$
\begin{aligned}
p & \approx\left|U_{e 2}\right|^{2} P_{H}+\left|U_{e 3}\right|^{2}\left(1-P_{H}\right) \\
& \approx \sin ^{2} \theta_{\odot} P_{H}+\left|U_{e 3}\right|^{2}\left(1-P_{H}\right) .
\end{aligned}
$$

Notice that, depending on the value of $P_{H}$, the $\nu_{e}$-survival probability takes the values between

$$
\left|U_{e 3}\right|^{2} \leqslant p \leqslant\left|U_{e 2}\right|^{2} \text {. }
$$

The fluxes at the earth are determined by Eq. (64), with $p$ and $\bar{p}$ given in Eqs. (88) and (87). Their features depend on the adiabaticity at the $H$ resonance, which is decided by the region in which $\left|U_{e 3}\right|^{2}$ lies.

Region I. The level crossing scheme (Fig. 5c) with adiabatic transitions in the $H$ resonance layer leads to the following transitions inside the star:

$$
\nu_{e} \rightarrow \nu_{3}, \quad \nu_{\mu^{\prime}} \rightarrow \nu_{1}, \quad \nu_{\tau^{\prime}} \rightarrow \nu_{2}
$$

Since $P_{H} \approx 0$, we get $p \approx\left|U_{e 3}\right|^{2}$, and the final spectra at the Earth:

$$
\begin{gathered}
F_{e} \approx\left|U_{e 3}\right|^{2} F_{e}^{0}+\left(1-\left|U_{e 3}\right|^{2}\right) F_{x}^{0} \approx F_{x}^{0}, \\
F_{e}^{-} \approx \cos ^{2} \theta_{\odot} F_{e}^{0}+\sin ^{2} \theta_{\odot} F_{x}^{0}, \\
4 F_{x} \approx F_{e}^{0}+\sin ^{2} \theta_{\odot} F_{e}^{0}+\left(2+\cos ^{2} \theta_{\odot}\right) F_{x}^{0} .
\end{gathered}
$$

The $\bar{\nu}_{e}$ spectrum is composite: this feature distinguishes the LMA scheme from the one with the SMA solution. The $\nu_{e}$ spectrum is hard, so $\left.\left\langle E_{\nu_{e}}\right\rangle\right\rangle\left\langle E_{\nu_{e}}\right\rangle$, as was the case with the SMA scheme with an adiabatic $H$ resonance.

No Earth matter effects are expected in the $\nu_{e}$ spectrum, since $P_{H} \approx 0$. At the same time, the $\bar{\nu}_{e}$ spectrum can show significant Earth matter effects. This is an important signature of this scenario.

Region II. The jump probability in the $H$ resonance is substantial and the following transitions occur:

$$
\nu_{e} \rightarrow \nu_{2}, \nu_{3}, \quad \nu_{\mu^{\prime}} \rightarrow \nu_{1}, \quad \nu_{\tau^{\prime}} \rightarrow \nu_{2}, \nu_{3} .
$$

The final spectra are determined by Eq. (64) with $p$ given by Eq. (88):

$$
p \approx \sin ^{2} \theta_{\odot} P_{H}
$$


Depending on $P_{H}$ and $\sin ^{2} \theta_{\odot} \approx\left|U_{e 2}\right|^{2} \approx 0.2-0.4$, the probability $p$ can take values between 0.02 and 0.4 .

The final spectra can be written as

$$
\begin{aligned}
F_{e} \approx & \sin ^{2} \theta_{\odot} P_{H} F_{e}^{0}+\left(1-\sin ^{2} \theta_{\odot} P_{H}\right) F_{x}^{0}, \\
F_{e}^{-} \approx & \cos ^{2} \theta_{\odot} F_{e}^{0}+\sin ^{2} \theta_{\odot} F_{x}^{0}, \\
4 F_{x} \approx & \left(1-\sin ^{2} \theta_{\odot} P_{H}\right) F_{e}^{0}+\sin ^{2} \theta_{\odot} F_{\bar{e}}^{0} \\
& +\left(3-\sin ^{2} \theta_{\odot}+\sin ^{2} \theta_{\odot} P_{H}\right) F_{x}^{0} .
\end{aligned}
$$

All the $\nu_{e}, \bar{\nu}_{e}$ and $\nu_{x}$ spectra are composite.

The Earth matter effects can be significant for both the $\nu_{e}$ and $\bar{\nu}_{e}$ spectra. The effects on the $\nu_{e}$ spectrum are suppressed moderately by a factor of $P_{H}$.

Region III. Here $P_{H} \approx 1$, so that according to Eq. (88), $p$ $\approx\left|U_{e 2}\right|^{2} \approx 0.2-0.4$. The $H$ resonance is inoperative, and the following transitions occur:

$$
\nu_{e} \rightarrow \nu_{2}, \quad \nu_{\mu^{\prime}} \rightarrow \nu_{1}, \quad \nu_{\tau^{\prime}} \rightarrow \nu_{3}
$$

Then we have $p=\sin ^{2} \theta_{\odot}$ and $\bar{p}=\cos ^{2} \theta_{\odot}$. All the three spectra- $\nu_{e}, \bar{\nu}_{e}$ and $\nu_{x}$-are composite.

Since $P_{H} \approx 1$, the Earth matter effects are expected to be significant for both $\nu_{e}$ and $\bar{\nu}_{e}$ spectra.

Let us consider in some details the Earth matter effects which can give important signatures of the LMA scheme. For the $\nu_{e}$ spectrum the effect can be significant in regions II and III. Inserting $P_{L}=0$ into Eq. (77), we get

$$
F_{e}^{D}-F_{e} \approx P_{H}\left(P_{2 e}-\left|U_{e 2}\right|^{2}\right)\left(F_{e}^{0}-F_{x}^{0}\right) .
$$

Using the constant density Earth approximation (77) we estimate that the factor $\left(P_{2 e}-\left|U_{e 2}\right|^{2}\right)$ can be as large as 0.3 in the energy range of $20-50 \mathrm{MeV}$.

Similarly, for the antineutrinos, we get

$$
F_{\bar{e}}^{D}-F_{\bar{e}}^{-} \approx\left(\bar{P}_{1 e}-\left|U_{e 1}\right|^{2}\right)\left(F_{\bar{e}}^{0}-F_{x}^{0}\right) .
$$

In the antineutrino channel, the effect can be significant in all three regions of $U_{e 3}$, since there is no $P_{H}$ suppression, and again the factor $\left(\bar{P}_{1 e}-\left|U_{e 1}\right|^{2}\right)$ can be as large as 0.3 for energies around $20-50 \mathrm{MeV}$.

In Fig. 9, we show the $\nu_{e}$ and $\bar{\nu}_{e}$ spectra for different distances traveled by the neutrinos through the Earth. The sign of the Earth effect (92),(93) is determined by the sign of $\left(F_{e}^{0}-F_{x}^{0}\right)$ in the neutrino channel and $\left(F_{e}^{0}-F_{x}^{0}\right)$ in the antineutrino channel. The effect changes sign at $E=E_{c}(E$ $=\bar{E}_{c}$ ) for the neutrinos (antineutrinos), as described in Sec. III C (Sec. III D). Since $P_{2 e} \geqslant\left|U_{e 2}\right|^{2}$ and $\bar{P}_{1 e} \geqslant\left|U_{e 1}\right|^{2}$, Eqs. (92) and (93) imply that the Earth effect results in the enhancement of neutrino (antineutrino) signal for $E<E_{c}$ ( $E$ $\left.<\bar{E}_{c}\right)$ and the depletion of the signal for $E>E_{c}\left(E>\bar{E}_{c}\right)$. Since the cross section of the neutrino detection interactions is larger at higher energies, a significant reduction in the $\nu_{e}$ $\left(\bar{\nu}_{e}\right)$ flux is expected at energies greater than $E_{c}\left(\bar{E}_{c}\right)$. It may

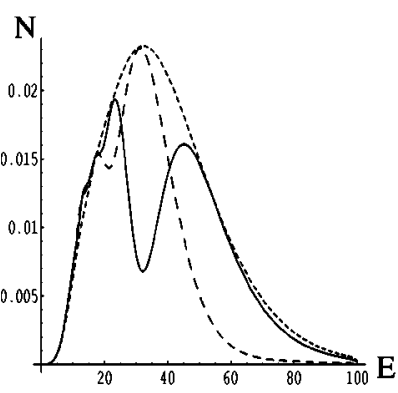

(a)

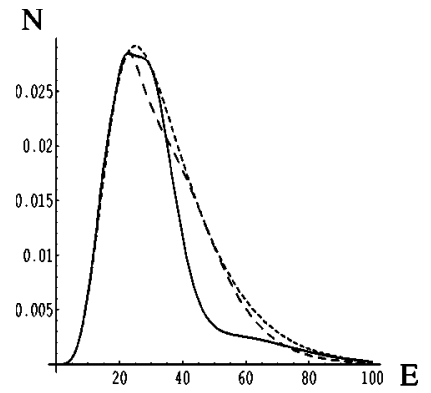

(b)
FIG. 9. The Earth matter effects on (a) the $\nu_{e}$ spectrum and (b) the $\bar{\nu}_{e}$ spectrum for $P_{H}=1$ in the scheme with the LMA solution $\left(\Delta m^{2}=2 \times 10^{-5} \mathrm{eV}^{2}, \sin ^{2} 2 \theta_{\odot}=0.9\right)$. The dotted, dashed and solid lines show the spectra of the number of $\nu-N$ charged current events when the distance travelled by the neutrinos through the earth is $d=0 \mathrm{~km}, d=4000 \mathrm{~km}$, and $d=6000 \mathrm{~km}$ respectively.

manifest itself as a dip in the final spectrum (see Fig. 9), and this spectral distortion can indicate the presence of significant Earth matter effects, even with the spectrum observed in only one detector.

\section{Scheme with the VO solution}

The mass and flavor spectrum for the scheme is shown in Fig. 10. The solar neutrino data are explained via the vacuum oscillations with $\Delta m^{2} \sim 10^{-10} \mathrm{eV}^{2}$ and a large mixing between $\nu_{e}$ and a combination of $\nu_{\mu}$ and $\nu_{\tau}$, Eq. (5).

The level crossing scheme is shown in Fig. 5c, where the resonance $L$ is close to $\rho=0$. It may occur in the neutrino or antineutrino channel. Depending on the details of the density profile in the star at low densities, it lies either in the nonadiabatic region (so that $P_{L}, \bar{P}_{L} \approx 1$ ) or in the transition region.

Let us first consider the antineutrino channel. According to the level crossing scheme, we get the transitions

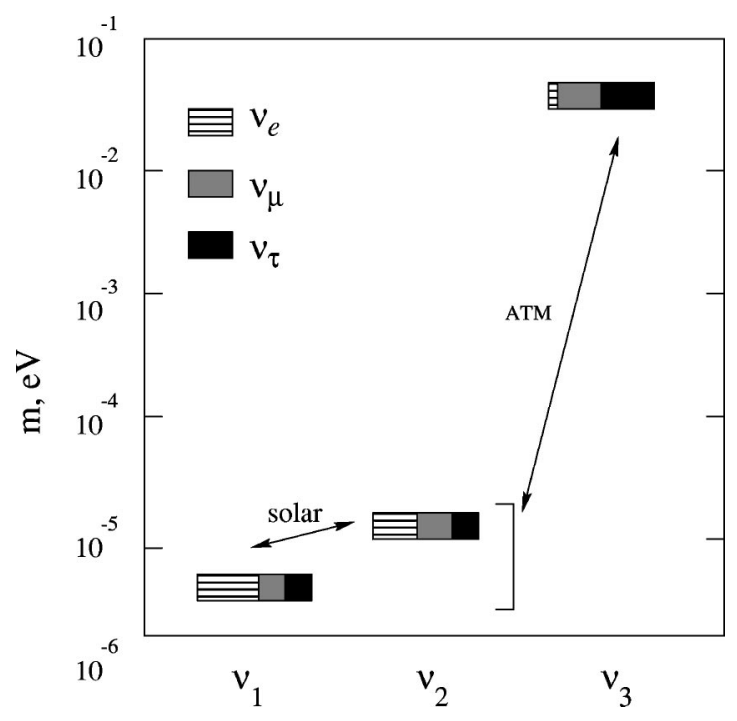

FIG. 10. Neutrino mass and mixing pattern of the scheme with the vacuum oscillation solution for the solar neutrino data. 


$$
\bar{\nu}_{e} \rightarrow \bar{\nu}_{1}, \bar{\nu}_{2}, \quad \bar{\nu}_{\mu^{\prime}} \rightarrow \bar{\nu}_{1}, \bar{\nu}_{2}, \quad \bar{\nu}_{\tau^{\prime}} \rightarrow \bar{\nu}_{3}
$$

The final fluxes are given by Eq. (55) and for $\bar{p}$ we get, from Eq. (60),

$$
\bar{p}=\cos ^{2} \theta_{\odot}\left(1-\bar{P}_{L}\right)+\sin ^{2} \theta_{\odot} \bar{P}_{L} .
$$

This implies that the value of $\bar{p}$ lies between $\cos ^{2} \theta_{\odot}$ and $\sin ^{2} \theta_{\odot}$, and the $\bar{\nu}_{e}$ spectrum is composite.

The fluxes in the neutrino channel depend on the region in which the oscillation parameters of the $H$ resonance lie.

Region $I$. The $H$ resonance is adiabatic, and the following transitions occur in the neutrino channel:

$$
\nu_{e} \rightarrow \nu_{3}, \quad \nu_{\mu^{\prime}} \rightarrow \nu_{1}, \nu_{2}, \quad \nu_{\tau^{\prime}} \rightarrow \nu_{1}, \nu_{2}
$$

Since $P_{H} \approx 0$, from Eq. (55) we get

$$
p \approx\left|U_{e 3}\right|^{2} \leqslant 0.03 \text {. }
$$

The $\nu_{e}$ then has the hard spectrum of the original $\nu_{x}$. The observed features of the final fluxes are the same as those in the scheme with the LMA solution and an adiabatic $H$ resonance (90).

The Earth matter effects are small for both the $\nu_{e}$ and $\bar{\nu}_{e}$ spectra, since the mixing angle $\bar{\theta}_{e 2}$ is suppressed in the Earth matter.

Region II. Jump probability in the $H$ resonance is substantial, so that the transitions inside the star are

$$
\nu_{e} \rightarrow \nu_{1}, \nu_{2}, \nu_{3}, \quad \nu_{\mu^{\prime}} \rightarrow \nu_{1}, \nu_{2}, \quad \nu_{\tau^{\prime}} \rightarrow \nu_{1}, \nu_{2}, \nu_{3}
$$

From Eq. (55), we can infer

$$
\left|U_{e 3}\right|^{2} \leqslant p \leqslant \cos ^{2} \theta_{\odot}
$$

where the lower bound corresponds to $P_{H}=0$, whereas the upper bound is for $P_{H}=P_{L}=1$. All the three spectra are then composite.

The Earth matter effects are small for both the $\nu_{e}$ and the $\bar{\nu}_{e}$ spectra.

Region III. Since the $H$ resonance is completely nonadiabatic, the neutrino transitions take place in $L$ resonance region only:

$$
\nu_{e} \rightarrow \nu_{1}, \nu_{2}, \quad \nu_{\mu^{\prime}} \rightarrow \nu_{1}, \nu_{2}, \quad \nu_{\tau^{\prime}} \rightarrow \nu_{3}
$$

From Eq. (55) we get, for $P_{H} \approx 1$,

$$
\begin{aligned}
p & \approx\left|U_{e 1}\right|^{2} P_{L}+\left|U_{e 2}\right|^{2}\left(1-P_{L}\right) \\
& \approx \cos ^{2} \theta_{\odot} P_{L}+\sin ^{2} \theta_{\odot}\left(1-P_{L}\right) .
\end{aligned}
$$

Thus,

$$
\sin ^{2} \theta_{\odot} \leqslant p \leqslant \cos ^{2} \theta_{\odot}
$$

and all the final spectra are composite.

The Earth matter effects are small for both the $\nu_{e}$ and the $\bar{\nu}_{e}$ spectra.

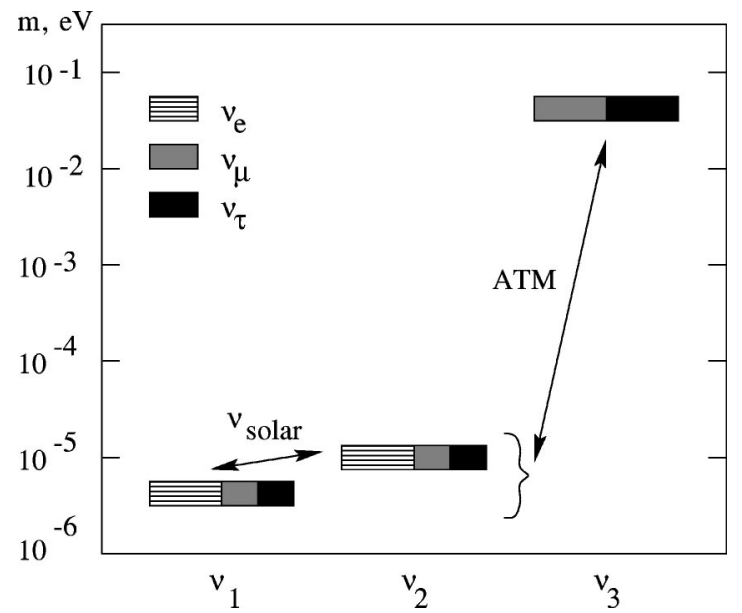

FIG. 11. The neutrino mass and mixing pattern of the bimaximal mixing scheme.

\section{Bi-maximal mixing scheme}

An extreme case of the VO scenario is the bimaximal mixing scheme [52]. The mass and flavor spectrum is as shown in Fig. 11. Here $\nu_{\mu}$ and $\nu_{\tau}$ mix maximally in $\nu_{\tau^{\prime}}$ $=\left(\nu_{\mu}+\nu_{\tau}\right) / \sqrt{2}$; in turn, the orthogonal combination $\nu_{\mu^{\prime}}$ $\equiv\left(\nu_{\mu}-\nu_{\tau}\right) / \sqrt{2}$ mixes maximally with $\nu_{e}$. In this scenario, $U_{e 3}=0$. The mass eigenstates are then

$$
\nu_{1}=\left(\nu_{e}-\nu_{\mu^{\prime}}\right) / \sqrt{2}, \quad \nu_{2}=\left(\nu_{e}+\nu_{\mu^{\prime}}\right) / \sqrt{2}, \quad \nu_{3}=\nu_{\tau^{\prime}} .
$$

The following transitions take place:

$$
\begin{array}{lll}
\nu_{e} \rightarrow \nu_{1}, \nu_{2}, & \nu_{\mu^{\prime}} \rightarrow \nu_{1}, \nu_{2}, & \nu_{\tau^{\prime}} \rightarrow \nu_{3}, \\
\bar{\nu}_{e} \rightarrow \bar{\nu}_{1}, \bar{\nu}_{2}, & \bar{\nu}_{\mu^{\prime}} \rightarrow \bar{\nu}_{1}, \bar{\nu}_{2}, \quad \bar{\nu}_{\tau^{\prime}} \rightarrow \bar{\nu}_{3} .
\end{array}
$$

The mass eigenstates $\nu_{1}$ and $\nu_{2}\left(\bar{\nu}_{1}\right.$ and $\left.\bar{\nu}_{2}\right)$ arriving at the Earth are observed with equal probability as electron or nonelectron neutrinos. The expected fluxes are then

$$
\begin{aligned}
F_{e} & =(1 / 2) F_{e}^{0}+(1 / 2) F_{x}^{0}, \\
F_{e}^{-} & =(1 / 2) F_{e}^{0}+(1 / 2) F_{x}^{0} \\
4 F_{x} & =(1 / 2) F_{e}^{0}+3 F_{x}^{0}+(1 / 2) F_{\bar{e}}^{0} .
\end{aligned}
$$

The $\nu_{e}$ and $\bar{\nu}_{e}$ spectra are composite with the soft and hard components in equal proportions. The $\bar{\nu}_{e}$ spectrum has a higher average energy than the $\nu_{e}$ spectrum, but the high energy tails of both the spectra are identical.

The Earth matter effects are small for both $\nu_{e}$ and $\bar{\nu}_{e}$ spectra due to the small value of $\Delta m_{\odot}^{2} \sim 10^{-10} \mathrm{eV}^{2}$.

\section{CONVERSION PROBABILITIES AND NEUTRINO FLUXES WITH THE INVERTED MASS HIERARCHY}

In the case of the inverted mass hierarchy (19), the mass eigenstates $\nu_{1}$ and $\nu_{2}$ are heavy and degenerate, whereas the third state $\nu_{3}$ is much lighter: $m_{1} \approx m_{2} \gg m_{3}$ (see e.g. Fig. 12 for the scenario with the SMA solution). 


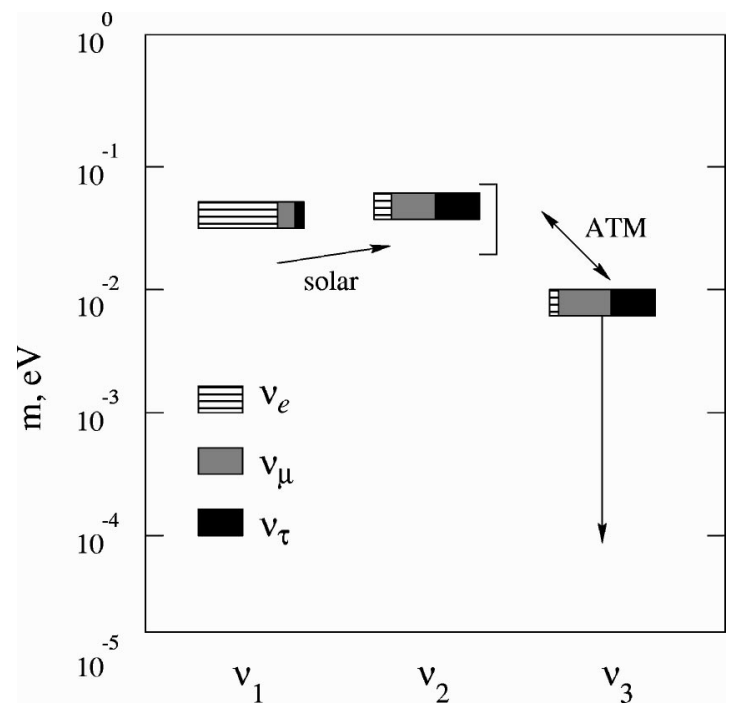

FIG. 12. The neutrino mass and mixing pattern for the scenario with inverted mass hierarchy and the SMA solution for the solar neutrino problem.

As shown in Figs. $5 \mathrm{~b}$ and $5 \mathrm{~d}$, the $H$ resonance is in the antineutrino channel, whereas the $L$ resonance lies in the neutrino channel for the SMA and LMA solutions. In the case of the VO solution, the $L$ resonance can be in either the neutrino or the antineutrino channel.

Like in the case of the normal mass hierarchy, the fluxes at the Earth can be written in terms of the survival probabilities $p$ and $\bar{p}$ of $\nu_{e}$ and $\bar{\nu}_{e}$ respectively, as given in Eq. (64). Let us find the expressions for the survival probabilities $p$ and $\bar{p}$.

As follows from the level crossing scheme (Figs. 5a and $5 \mathrm{~d})$, the matter eigenstates in the high density region ( $\rho$ $\left.\gg \rho_{H}, \rho_{L}\right)$ are

$$
\begin{aligned}
& \nu_{1 m}=\nu_{\mu^{\prime}}, \quad \nu_{2 m}=\nu_{e}, \quad \nu_{3 m}=\nu_{\tau^{\prime}}, \\
& \bar{\nu}_{1 m}=\bar{\nu}_{\tau^{\prime}}, \quad \bar{\nu}_{2 m}=\bar{\nu}_{\mu^{\prime}}, \quad \bar{\nu}_{3 m}=\bar{\nu}_{e} .
\end{aligned}
$$

The state $\nu_{e}$, which coincides with $\nu_{2 m}$ in the production region, crosses the $H$-resonance layer adiabatically (since the resonance is in the antineutrino channel and the mixing in the neutrino channel is small), and reaches the $L$ layer as $\nu_{2 m}$. It reaches the surface of the Earth as $\nu_{1}$ with the probability $P_{L}$ and as $\nu_{2}$ with the probability $\left(1-P_{L}\right)$. Then

$$
p=\left|U_{e 1}\right|^{2} P_{L}+\left|U_{e 2}\right|^{2}\left(1-P_{L}\right) \text {. }
$$

The state $\bar{\nu}_{e}$, which coincides with $\bar{\nu}_{3 m}$ in the production region, flips to $\bar{\nu}_{1 m}$ in the resonance region $H$ with the probability $\bar{P}_{L}$. Depending on the parameters of the solar neutrino solution, the propagation of the antineutrinos in the $L$-resonance region (i.e. near $\rho=0$ ) may or may not be adiabatic which is described by $\bar{P}_{L}$. Computing the probabilities of transitions of $\bar{\nu}_{e}$ to $\bar{\nu}_{1}, \bar{\nu}_{2}, \bar{\nu}_{3}$ and performing projection of the result back onto $\bar{\nu}_{e}$ back we get [similarly to Eq. (55)] the survival probability $\bar{p}$ for the $\bar{\nu}_{e}$ :

$$
\bar{p}=\left|U_{e 1}\right|^{2} \bar{P}_{H}\left(1-\bar{P}_{L}\right)+\left|U_{e 2}\right|^{2} \bar{P}_{H} \bar{P}_{L}+\left|U_{e 3}\right|^{2}\left(1-\bar{P}_{H}\right) .
$$

[Here we have also averaged out the interference effects between the mass eigenstates, so that the three terms in Eq. (102) correspond to the three mass eigenstates.]

Let us consider the Earth matter effects on the $\nu_{e}$ and $\bar{\nu}_{e}$ spectra for the inverted mass hierarchy. Since there is no $H$ resonance in the neutrino channel, the Earth effects for the inverted hierarchy are the same as those described for the normal mass hierarchy (see Sec. III C) with $P_{H}=1$ :

$$
F_{e}^{D 1}-F_{e}^{D 2} \approx\left(P_{2 e}^{(1)}-P_{2 e}^{(2)}\right)\left(1-2 P_{L}\right)\left(F_{e}^{0}-F_{x}^{0}\right) .
$$

The matter effects are significant for the scheme with the LMA solution. The suppression factor of $P_{H}$ [which was present for the normal mass hierarchy—see Eq. (75)] is now absent, so the Earth matter effects can be larger than in the case of the normal mass hierarchy (see Fig. 9a).

The Earth matter effects on the antineutrino spectra can be calculated as follows. Using the same arguments as for Eq. (102), we find the survival probability of $\bar{\nu}_{e}$ at the detector:

$$
\bar{p}^{D}=\bar{P}_{1 e} \bar{P}_{H}\left(1-\bar{P}_{L}\right)+\bar{P}_{2 e} \bar{P}_{H} \bar{P}_{L}+\bar{P}_{3 e}\left(1-\bar{P}_{H}\right) .
$$

Then Eqs. (102) and (104) give

$$
\begin{aligned}
\bar{p}^{D}-\bar{p}= & \left(\bar{P}_{1 e}-\left|U_{e 1}\right|^{2}\right)\left(1-2 \bar{P}_{L}\right) \bar{P}_{H} \\
& +\left(\bar{P}_{3 e}-\left|U_{e 3}\right|^{2}\right)\left(1-\bar{P}_{H}-\bar{P}_{H} \bar{P}_{L}\right),
\end{aligned}
$$

where we have used $\Sigma \bar{P}_{i e}=1$. Since $\bar{\nu}_{3}$ oscillates inside the Earth with a very small depth [inequality (73) is valid with $P_{3 e}$ replaced by $\left.\bar{P}_{3 e}\right]$, the second term in Eq. (105) can be neglected. Therefore, finally we get

$$
F_{\bar{e}}^{D 1}-F_{\bar{e}}^{D 2} \approx \bar{P}_{H}\left(\bar{P}_{1 e}^{(1)}-\bar{P}_{1 e}^{(2)}\right)\left(1-2 \bar{P}_{L}\right)\left(F_{\bar{e}}^{0}-F_{x}^{0}\right) .
$$

The Earth matter effects on the $\bar{\nu}_{e}$ spectrum for the inverted hierarchy are then the same as those described for the normal hierarchy in Sec. III C, but further suppressed by a factor of $\bar{P}_{H}$. Significant effects can be observed only with the LMA scenario and when the $H$ resonance is nonadiabatic.

The Earth matter effects on both $\nu_{e}$ and $\bar{\nu}_{e}$ spectra are factorized, as in the case of the normal mass hierarchy. They are proportional to (i) the difference in original $\nu_{e}\left(\bar{\nu}_{e}\right)$ and $\nu_{x}$ fluxes, (ii) a factor which determines the conversion inside the star, and (iii) the difference of the oscillation probabilities inside the Earth.

Notice that the expressions for the Earth matter effects in the inverted mass hierarchy case, Eqs. (103),(106), have the same form as the expressions in the normal hierarchy case, Eqs. (75),(80), with the substitution $\nu \leftrightarrow \bar{\nu}, P_{H} \leftrightarrow \bar{P}_{H}$. 
Let us now calculate the values of the survival probabilities $p$ and $\bar{p}$ for the three scenarios with the SMA, LMA and VO solutions for the solar neutrino problem respectively. Within each scenario, the final spectra would depend on the adiabaticity of antineutrino conversions in the $H$ resonance layer, i.e. on the value of $\left|U_{e 3}\right|^{2}$.

We shall consider the effects of neutrino conversions in three possible regions of $\left|U_{e 3}\right|^{2}$ : regions I, II and III as described in Sec. II E. When $\left|U_{e 3}\right|^{2}$ lies in region III, i.e. when the $H$ resonance is inoperative, the resulting final spectra for a given solar neutrino solution are the same as those obtained in Secs. IV A, IV B and IV C for the normal mass hierarchy with the same mixing parameters. Therefore, we need to consider only the cases when $\left|U_{e 3}\right|^{2}$ lies in region I or II.

\section{A. Scheme with the SMA solution}

The characteristics of the $\nu_{e}$ spectrum are independent of the conversions at the $H$ resonance layer [see Eq. (101)]. The $L$ resonance is in the transition region so that partial jumps from one level to another occur. As a consequence, from the level crossing scheme (Fig. 5b), we get the following transitions:

$$
\nu_{e} \rightarrow \nu_{1}, \nu_{2}, \quad \nu_{\mu^{\prime}} \rightarrow \nu_{1}, \nu_{2}, \quad \nu_{\tau^{\prime}} \rightarrow \nu_{3} .
$$

Neglecting terms proportional to the small $\left|U_{e 2}\right|^{2}$ in Eq. (101), we get

$$
p \approx P_{L} .
$$

The $\nu_{e}$ spectrum is thus composite.

Let us now examine the final $\bar{\nu}_{e}$ spectrum. Since the $L$ resonance is in the neutrino channel, the antineutrino transitions in the $L$ resonance layer are adiabatic, so that $\bar{P}_{L} \approx 0$. The characteristics of the final $\bar{\nu}_{e}$ spectrum depend on the region in which the value of $\left|U_{e 3}\right|^{2}$ lies.

Region I. The following transitions take place inside the star:

$$
\bar{\nu}_{e} \rightarrow \bar{\nu}_{3}, \quad \bar{\nu}_{\mu^{\prime}} \rightarrow \bar{\nu}_{2}, \quad \bar{\nu}_{\tau^{\prime}} \rightarrow \bar{\nu}_{1} .
$$

For $P_{H} \approx 0$, Eq. (102) gives

$$
\bar{p} \approx\left|U_{e 3}\right|^{2}<0.03 \text {. }
$$

The final $\bar{\nu}_{e}$ spectrum is then almost completely the original hard spectrum of $\nu_{x}$ :

$$
F_{e}^{-} \approx F_{x}^{0} .
$$

The $\bar{\nu}_{e}$ spectrum is practically the same as the original hard spectrum of $\nu_{x}$, whereas the final $\nu_{e}$ and $\nu_{x}$ spectra are composite.

The Earth matter effects on the $\nu_{e}$ spectrum (103) are small, $\leqslant 10 \%$ (see Sec. IV A), but still may be observable. The Earth matter effects on the $\bar{\nu}_{e}$ spectrum (106) are negli- gible. They are suppressed by the factor of $\bar{P}_{H} \approx 0$ and further by the small mixing of the antineutrinos in the earth matter.

Region II. Taking into account jumps of states at $H$ resonance we get the following transitions:

$$
\bar{\nu}_{e} \rightarrow \bar{\nu}_{1}, \bar{\nu}_{3}, \quad \bar{\nu}_{\mu^{\prime}} \rightarrow \bar{\nu}_{2}, \quad \bar{\nu}_{\tau^{\prime}} \rightarrow \bar{\nu}_{1}, \bar{\nu}_{3} .
$$

Inserting $\bar{P}_{L} \approx 0$ in Eq. (102), we find

$$
\bar{p} \approx \bar{P}_{H} \cos ^{2} \theta_{\odot} \approx \bar{P}_{H}
$$

All the three spectra are then composite.

The Earth matter effects (103) are small, $\$ 10 \%$ (see Sec. IV A) for $\nu_{e}$ and negligible for the $\bar{\nu}_{e}$.

\section{B. Scheme with the LMA solution}

The neutrino transitions at the $L$ resonance are adiabatic, so that the following transitions take place according to the level crossing scheme (Fig. 5d):

$$
\nu_{e} \rightarrow \nu_{2}, \quad \nu_{\mu^{\prime}} \rightarrow \nu_{1}, \quad \nu_{\tau^{\prime}} \rightarrow \nu_{3} .
$$

Inserting $P_{L} \approx 0$ in Eq. (101), we get

$$
p \approx\left|U_{e 2}\right|^{2} \approx \sin ^{2} \theta_{\odot} \sim 0.2-0.4,
$$

and consequently, the $\nu_{e}$ spectrum is composite:

$$
F_{e} \approx \sin ^{2} \theta_{\odot} F_{e}^{0}+\cos ^{2} \theta_{\odot} F_{x}^{0} .
$$

Let us examine the final $\bar{\nu}_{e}$ spectrum. The antineutrino transitions in the $L$ resonance region are also adiabatic, so that $\bar{P}_{L} \approx 0$. Conversions in the $H$ resonance region, and therefore the characteristics of the final $\bar{\nu}_{e}$ spectrum depend on $\left|U_{e 3}\right|^{2}$.

Region $I$. Here the $H$ resonance is adiabatic and we get the following transitions:

$$
\bar{\nu}_{e} \rightarrow \bar{\nu}_{3}, \quad \bar{\nu}_{\mu^{\prime}} \rightarrow \bar{\nu}_{2}, \quad \bar{\nu}_{\tau^{\prime}} \rightarrow \bar{\nu}_{1} .
$$

Since $P_{H} \approx 0$, Eq. (102) gives

$$
\bar{p} \approx\left|U_{e 3}\right|^{2}<0.03,
$$

and consequently, the $\bar{\nu}_{e}$ spectrum is then almost completely the original hard spectrum of $\nu_{x}$ :

$$
F_{e}^{-} \approx F_{x}^{0} .
$$

The features of the final spectra are the same as those of the scheme with the inverted mass hierarchy and SMA solution (with $\left|U_{e 3}\right|^{2}$ in region I).

The Earth matter effects on the $\nu_{e}$ spectrum (103) can be significant, as can be seen from Fig. 9a. In contrast, the Earth effects on the $\bar{\nu}_{e}$ spectrum (106) are negligible: they are suppressed by the factor of $\bar{P}_{H} \approx 0$ and by the small mixing of the antineutrinos in the Earth matter. 
Region II. The transitions in the $H$ resonance layer are incomplete and therefore according to the level crossing scheme we have

$$
\bar{\nu}_{e} \rightarrow \bar{\nu}_{1}, \bar{\nu}_{3}, \quad \bar{\nu}_{\mu^{\prime}} \rightarrow \bar{\nu}_{2}, \quad \bar{\nu}_{\tau^{\prime}} \rightarrow \bar{\nu}_{1}, \bar{\nu}_{3}
$$

Inserting $\bar{P}_{L} \approx 0$ in Eq. (102), we get

$$
\bar{p} \approx \bar{P}_{H}\left|U_{e 1}\right|^{2}=\bar{P}_{H} \cos ^{2} \theta_{\odot} .
$$

The features of the final spectra are the same as those of the scheme with the inverted mass hierarchy and SMA solution (with $\left|U_{e 3}\right|^{2}$ in region II).

The Earth matter effects can be significant both in the $\nu_{e}$, Eq. (103), and in the $\bar{\nu}_{e}$, Eq. (106), channels (see Fig. 9).

\section{Scheme with the VO solution}

The conversions in the $H$ resonance layer does not influence $\nu_{e}$ flux. In the $L$ resonance layer the adiabaticity is moderately broken: $P_{L} \neq 0$. From the level crossing scheme (Fig. 5d), the transitions taking place inside the star are

$$
\nu_{e} \rightarrow \nu_{1}, \nu_{2}, \quad \nu_{\mu^{\prime}} \rightarrow \nu_{1}, \nu_{2}, \quad \nu_{\tau^{\prime}} \rightarrow \nu_{3} .
$$

From Eq. (101), we get

$$
\sin ^{2} \theta_{\odot}<p<\cos ^{2} \theta_{\odot}
$$

and since $\sin ^{2} \theta_{\odot}>0.3$, we get $p=0.3-0.7$. The $\nu_{e}$ spectrum is thus composite.

Let us now examine the final $\bar{\nu}_{e}$ spectrum.

Region I. Because of adiabaticity in the $H$ resonance layer, the following transitions take place:

$$
\bar{\nu}_{e} \rightarrow \bar{\nu}_{3}, \quad \bar{\nu}_{\mu^{\prime}} \rightarrow \bar{\nu}_{1}, \bar{\nu}_{2}, \quad \bar{\nu}_{\tau^{\prime}} \rightarrow \bar{\nu}_{1}, \bar{\nu}_{2}
$$

Here $P_{H} \approx 0$, and from Eq. (102) we get

$$
\bar{p} \approx\left|U_{e 3}\right|^{2}<0.03 \text {. }
$$

The $\bar{\nu}_{e}$ spectrum is then almost completely the original hard spectrum of $\nu_{x}$ :

$$
F_{\bar{e}} \approx F_{x}^{0}
$$

The features of the final spectra are similar to those of the scheme with the inverted mass hierarchy and SMA solution (with $\left|U_{e 3}\right|^{2}$ in region I): the $\nu_{e}$ spectrum is composite whereas the $\bar{\nu}_{e}$ spectrum is purely hard.

The earth matter effects on both $\nu_{e}$ and $\bar{\nu}_{e}$ spectra are negligible.

Region II. The adiabaticity is moderately broken both in the $H$ and in the $L$ resonance layers and the transitions proceed as

$$
\bar{\nu}_{e} \rightarrow \bar{\nu}_{1}, \bar{\nu}_{2}, \bar{\nu}_{3}, \quad \bar{\nu}_{\mu^{\prime}} \rightarrow \bar{\nu}_{1}, \bar{\nu}_{2}, \quad \bar{\nu}_{\tau^{\prime}} \rightarrow \bar{\nu}_{1}, \bar{\nu}_{2}, \bar{\nu}_{3}
$$

Correspondingly Eq. (102) gives

$$
\bar{p} \approx \bar{P}_{H}\left[\cos ^{2} \theta_{\odot}\left(1-\bar{P}_{L}\right)+\sin ^{2} \theta_{\odot} \bar{P}_{L}\right] .
$$

The features of the final spectra are the same as those of the scheme with the inverted mass hierarchy and SMA solution (with $\left|U_{e 3}\right|^{2}$ in region II).

The Earth matter effects on both $\nu_{e}$ and $\bar{\nu}_{e}$ spectra are negligible.

Let us summarize the results. Some salient features of the final spectra with the inverted mass hierarchy are the following:

(i) The neutronization peak contains both $\nu_{e}$ and $\nu_{x}$ fluxes, in all the scenarios.

(ii) The final $\nu_{e}$ spectrum is composite in all scenarios. The characteristics of this spectrum are independent of the oscillation parameters in the $H$ resonance region.

(iii) The final $\nu_{x}$ spectrum is composite in all scenarios.

(iv) When the value of $\left|U_{e 3}\right|^{2}$ lies in region I, the final $\bar{\nu}_{e}$ spectrum is almost the original hard spectrum of $\nu_{x}$. For $\left|U_{e 3}\right|^{2}$ in regions II and III the $\bar{\nu}_{e}$ spectrum is composite.

(v) The Earth matter effects on the $\nu_{e}$ spectrum can be significant for the scheme with the LMA solution, marginally observable for the scheme with the SMA solution and negligible for the VO solution.

(vi) The Earth matter effects on the $\bar{\nu}_{e}$ spectrum can be significant only for the scheme with the LMA solution, and when the antineutrino transitions at the $H$ resonance are not adiabatic. In all the other scenarios, the earth matter effects on $\bar{\nu}_{e}$ spectrum are negligible.

(vii) When the value of $\left|U_{e 3}\right|^{2}$ lies in region III, the final spectra (including the Earth matter effects on them) are indistinguishable from those obtained with the scheme having the same oscillation parameters but the normal mass hierarchy.

\section{SIGNALS OF MIXING AND SIGNATURES OF MIXING SCHEMES}

The future detection of a supernova neutrino burst by the underground neutrino detectors has been discussed in [5356]. For a typical supernova at $10 \mathrm{kpc}$, about $5000 \bar{\nu}_{e}$ events are expected to be detected at SK and a few hundred events each in SNO [57], LVD [58] and MACRO [59]. These detectors can reconstruct the energy of the outgoing charged lepton from the $\bar{\nu}_{e}-p$ charged current interactions. SK and SNO can also observe the direction of the charged lepton. In addition, SNO can detect $\nu_{e}$ and reconstruct its energy through the $\nu_{e}-d$ charged current interaction inside the $D_{2} \mathrm{O}$. The feasibility of measuring the absolute values of the neutrino masses through the time-of-flight delays has been studied in [13,60-62]. Here, we concentrate on the features of the final neutrino spectra that are relevant for identification of the neutrino mass and flavor spectrum and elaborate on the effects that can be observed in the earth detectors.

\section{A. Neutronization peak}

The neutronization peak can be identified as the burst in the neutrino signal during the first few milliseconds. If there 
is no neutrino mixing, the neutrinos will be predominantly $\nu_{e}$. Let the expected ratio of the number of charged current (CC) events to the number of neutral current (NC) events during the burst be

$$
R_{0}^{B} \equiv \frac{N^{C C}}{N^{N C}}
$$

If neutrino conversions transform some $\nu_{e}$ into $\nu_{x}$, the number of charged current events decreases while the number of neutral current events remains unchanged. Then, the observed value of this ratio is $R^{B}<R_{0}^{B}$.

When the value of $\left|U_{e 3}\right|^{2}$ lies in the region I in the case of the normal mass hierarchy (so that the neutrino transitions in the $H$ resonance layer are adiabatic), the $\nu_{e}$ flux, and hence the number of charged current events, gets suppressed by a factor of $\approx\left|U_{e 3}\right|^{2}$ (see Secs. IV A, IV B, IV C):

$$
\frac{R^{B}}{R_{0}^{B}} \approx\left|U_{e 3}\right|^{2} \leqslant 0.03 .
$$

The observation of $R_{B}>0.03 R_{0}^{B}$ would then point against $\left|U_{e 3}\right|^{2}$ in region I for the normal mass hierarchy. According to Eq. (40), this will allow us to put an upper bound on the mixing parameter $\left|U_{e 3}\right|^{2}$ :

$$
\left|U_{e 3}\right|^{2} \lesssim 3 \times 10^{-4} .
$$

\section{B. $\langle E\rangle$ inequalities and high energy "tails"}

In the absence of mixing, one should observe $\left\langle E_{\nu_{e}}\right\rangle$ $\left\langle\left\langle E_{\bar{\nu}_{e}}\right\rangle\right.$. The inversion of this inequality, i.e. $\left.\left\langle E_{\nu_{e}}\right\rangle\right\rangle\left\langle E_{\bar{\nu}_{e}}\right\rangle$, is the signature of the $\nu$ conversion. It implies that the contribution of the converted original hard $\nu_{x}$ spectrum to the final $\nu_{e}$ flux is significantly larger than its contribution to the final $\bar{\nu}_{e}$ flux.

In the case of the normal mass hierarchy, the inverted inequality for $\langle E\rangle$ will be observed when the transitions in the $H$ resonance layer are adiabatic. The observation of the normal inequality $\left\langle E_{\nu_{e}}\right\rangle\left\langle\left\langle E_{\nu_{e}}\right\rangle\right.$ can exclude this scenario.

In the case of inverted mass hierarchy, the adiabatic transitions in the $H$ resonance layer preserve the original inequalities for $\langle E\rangle:\left\langle E_{\nu_{e}}\right\rangle\left\langle\left\langle E_{\nu_{e}}\right\rangle\right.$. Therefore, an observation of the inequality $\left\langle E_{\nu_{e}}\right\rangle>\left\langle E_{\nu_{e}}\right\rangle$ rules out the scenarios which have the inverted mass hierarchy with the value of $\left|U_{e 3}\right|^{2}$ in region I. If the mass hierarchy is known to be the inverted one, an upper bound (108) on the mixing parameter $\left|U_{e 3}\right|^{2}$ can be obtained.

The relative difference of the $\nu_{e}, \bar{\nu}_{e}$ and $\nu_{x}$ spectra is especially significant in the high energy ends ("tails") of the spectra, where the fluxes decrease exponentially with the increase of energy. In the absence of mixing, one expects

$$
\frac{N_{e}\left(E>E_{\text {tail }}\right)}{N_{e}^{-}\left(E>E_{\text {tail }}\right)} \ll 1, \quad \frac{N_{e}^{-}\left(E>E_{\text {tail }}\right)}{N_{x}\left(E>E_{\text {tail }}\right)} \ll 1
$$

for high enough energy $E_{\text {tail }}$ (energy greater than the peak energies of the spectra). Here $N_{i}\left(E>E_{\text {tail }}\right)$ is the integrated number of $\nu_{i}-N$ charged current events above the energy $E_{\text {tail }}$.

The observation of the high energy ends of the $\nu_{e}$ and $\bar{\nu}_{e}$ spectra, where the contributions due to the original $\nu_{e}$ and $\bar{\nu}_{e}$ fluxes would be negligible, allows one to measure the contribution of the original hard $\nu_{x}$ spectrum to the final $\nu_{e}$ and $\bar{\nu}_{e}$ spectra.

Important conclusions can be drawn from the studies of the ratio

$$
R\left(E_{\text {tail }}\right) \equiv \frac{N_{e}\left(E>E_{\text {tail }}\right)}{N_{e}^{-}\left(E>E_{\text {tail }}\right)}
$$

as $E_{\text {tail }}$ increases.

(1) $R\left(E_{\text {tail }}\right) \rightarrow \infty$. This indicates that the tail of the $\nu_{e}$ spectrum extends to significantly higher energies than that of the $\bar{\nu}_{e}$ spectrum. This will testify for $\nu_{e} \leftrightarrow \nu_{x}$ conversions. Moreover, it will be a clear signal that the $\bar{\nu}_{e} \leftrightarrow \nu_{x}$ conversions are negligible; i.e., the $\bar{\nu}_{e}$ spectrum is the original soft spectrum. This would be an indication of (i) the SMA solution in the conventional hierarchy (Sec. IV A) or (ii) highly non-adiabatic $H$ transitions with the SMA solution in the inverted hierarchy (Sec. V A). If the mass hierarchy is known to be an inverted one, then this signal indicates that the value of $\left|U_{e 3}\right|^{2}$ lies in region III. From Eq. (41), we obtain a very strong bound on the parameter $\left|U_{e 3}\right|^{2}$ :

$$
4\left|U_{e 3}\right|^{2} \lesssim 10^{-5} \text {. }
$$

(2) $R\left(E_{\text {tail }}\right) \rightarrow 0$. Notice that the limit $R\left(E_{\text {tail }}\right) \rightarrow 0$ would be true in the absense of any neutrino conversion at all or in the absence of conversion of the electron neutrinos. This situation is not realized in any of the scenarios we have discussed. The observation of $R\left(E_{\text {tail }}\right) \rightarrow 0$ would exclude all the scenarios under consideration and thus testify for the solution of the solar neutrino problem which differs from the MSW or VO solutions. Another possibility could be related to a compact star with a very sharp density profile, so that the $L$ resonance is in the non-adiabatic region. In this case, the solar solution has to be SMA and the $H$ resonance has to be inoperative for neutrinos.

(3) $R\left(E_{\text {tail }}\right) \rightarrow$ const. This would testify for the contribution of the original $\nu_{x}$ to both final $\nu_{e}$ and $\bar{\nu}_{e}$ spectra. Moreover, the value of the constant will give us information about the mixing parameters.

In principle, the spectra of $\nu_{x}$ can be reconstructed by having a series of neutral current detectors with different thresholds. The $\nu_{x}$ spectrum will always have a dominating hard component. Therefore, the comparison of the high energy tails of the $\nu_{e}, \bar{\nu}_{e}$ with that of $\nu_{x}$ can directly give the measure of the hard component of the $\nu_{e}, \bar{\nu}_{e}$ spectra. The ability to measure the $\nu_{x}$ spectrum would also be useful in order to check for the presence of sterile neutrinos [9]. 


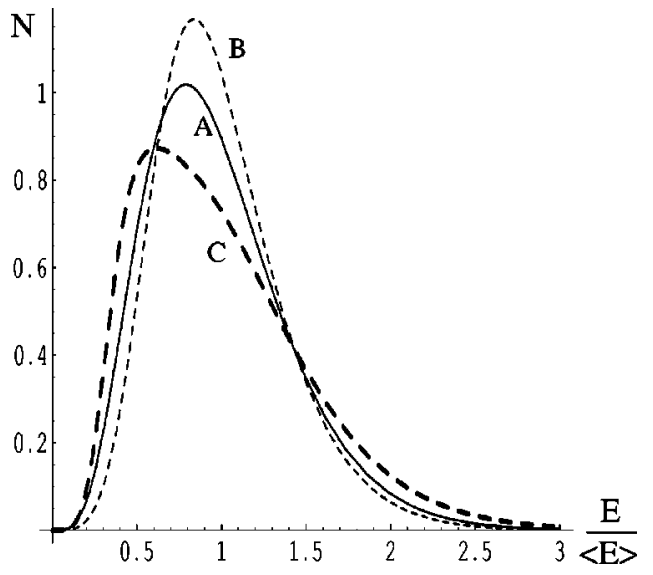

FIG. 13. The number of $\nu-N$ charged current events for (A) a Fermi-Dirac spectrum $(T=3, \eta=0)$ (the solid line), (B) a pinched spectrum $(T=3, \eta=3)$ (the dotted line), and (C) a mixture of two pinched spectra $\left(T=3, \eta=3\right.$ and $T=8, \eta=1$ with $\left.E_{n a}=5 \mathrm{MeV}\right)$ (the dashed line).

\section{Identification of a composite spectrum}

The final $\nu_{e}\left(\bar{\nu}_{e}\right)$ spectrum can be qualitatively divided into three types: (a) the original "soft" spectrum of the $\nu_{e}$ $\left(\bar{\nu}_{e}\right)$ [corresponding to the survival probability $p=1(\bar{p}$ $=1$ ) which would be the case in the absence of conversion], (b) the "hard" original spectrum of $\nu_{x}$ [corresponding to the survival probability $p=0(\bar{p}=0)$ which would be the case when there is a complete interchange of spectra], and (c) the "composite" spectrum, which is a mixture of the original soft and the hard spectra in comparable proportions.

In order to distinguish between (a) and (b), the values of "temperatures" (or average energies) of the original spectra need to be known or the $\nu_{e}$ and $\bar{\nu}_{e}$ spectra have to be compared (as in Sec. VI B). A composite spectrum may be identified without the knowledge of the initial temperatures and independently of the observation of the other spectrum, through the "broadening" phenomenon.

As described in Sec. II A, the instantaneous original neutrino spectra have a narrower energy distribution than the Fermi-Dirac one (see Fig. 1). The mixing between two neutrino species with different mean energies gives rise to an effective broadening of the spectrum (see Fig. 13), so that the final spectrum need not be pinched even though the two individual original spectra were pinched. In other words, the effective $\eta$ for a mixed spectrum may be negative even though the individual $\eta_{i}$ 's of the constituent spectra were positive. The broadening of the spectrum can be checked by fitting the final spectrum with the parameters $T_{i}$ and $\eta_{i}$, Eq. (7), and establishing the sign of $\eta_{i}$.

Note that the "pinching" phenomenon is established in a model independent manner only for a constant mean energy of the spectrum. A considerable variation in the average energy of neutrinos of a given species during the cooling phase could be responsible for the broadening of the time integrated spectrum even in the absence of mixing. Therefore, in order to establish the broadening due to mixing, it is crucial to study the spectra in short intervals of time.
Clearly, it will be difficult to observe broadening of spectra, if the mean energies of the original spectra are very close, or if one of the original spectrum dominates in the final mixed spectrum.

The observation of broadening of the $\nu_{e}$ spectrum would point against an adiabatic $H$ resonance in the normal hierarchy (see Secs. IV A, IV B, IV C). This would imply a strong upper bound (108) on the value of $\left|U_{e 3}\right|^{2}$.

The observation of broadening of the $\bar{\nu}_{e}$ spectrum would be a strong indication against

(i) the SMA solution with the normal hierarchy (Sec. IV A),

(ii) completely adiabatic $H$ transitions in the case of inverted hierarchy (Secs. V A, V B, V C), and

(iii) highly non-adiabatic $H$ transitions for the SMA solution with the inverted hierarchy (Sec. V A).

In particular, if the mass hierarchy is inverted, the broadening of $\bar{\nu}_{e}$ spectrum indicates that the $H$ resonance is not completely adiabatic. This gives a strong upper bound (108) on $\left|U_{e 3}\right|^{2}$.

If both the $\nu_{e}$ and $\bar{\nu}_{e}$ spectra are established to be composite, then the upper bound (108) on $\left|U_{e 3}\right|^{2}$ holds irrespective of the type of mass hierarchy.

\section{Earth matter effects}

The Earth matter effects can be observed through the comparison between the spectra at two detectors or through the studies of the distortion of a spectrum in one detector (see Figs. 7 and 9).

The Earth matter effects are significant in the neutrino channel only in the cases of (i) normal mass hierarchy with the SMA or LMA scenario and a non-adiabatic $H$ resonance, (ii) inverted mass hierarchy with the SMA or LMA scenario. In the antineutrino channels, significant Earth matter effects are observed (Sec. III D) only with (i) normal mass hierarchy with the LMA scenario, (ii) inverted mass hierarchy with LMA scenario and a non-adiabatic $H$ resonance.

\section{IDENTIFICATION OF THE MASS SPECTRUM}

We show that with the $3 \nu$ schemes which explain the solar and atmospheric neutrino data, one can make rather reliable predictions of the conversion effects for supernova neutrinos. The predictions differ for different schemes, which opens up the possibility of discriminating among them using the data from the neutrino bursts.

We have studied the manifestation of the conversion effects in (i) the flavor composition of the neutronization peak: the main effect here is the partial or complete change of the flavor of the peak. This can be established by the comparison of the fluxes detected by charged current and neutral current interactions during the neutronization burst. (ii) Modifications of the $\nu_{e}$ and $\bar{\nu}_{e}$ spectra: here one expects an appearance of hard or composite $\nu_{e}$ and/or $\bar{\nu}_{e}$ spectra due to mixing, instead of their original soft spectra. One can identify these effects of mixing by studying the average energies of 
spectra, the tails of spectra, and searching for the widening of the spectra. (iii) Earth matter effects: this can be done by detailed studies of the shapes of the energy spectra or by the comparison of signals observed in different detectors.

The final spectra of $\nu_{e}$ and $\bar{\nu}_{e}$ can be characterized by the values of the survival probabilities $p$ and $\bar{p}$ respectively. The conversion probabilities depend significantly on the value of $\left|U_{e 3}\right|^{2}$. We have divided the whole range of possible values of $\left|U_{e 3}\right|^{2}$ in three regions I, II, and III, and have made definite predictions in each of these regions. Table I gives the values of the survival probabilities for the neutrino mass spectra under discussion.

The qualitative features of the final neutrino fluxes for various neutrino mass spectra, obtained in Secs. III-V, are summarized in Table II. Let us first make some general observations from the table.

(1) The complete disappearance of the $\nu_{e}$ neutronization peak (strong change of its flavor) and a pure hard spectrum of $\nu_{e}$ during the cooling stage always appear together. Their observation will testify for $\left|U_{e 3}\right|^{2}$ in the region I and normal mass hierarchy, irrespective of the solution of the solar neutrino problem.

(2) A pure hard $\bar{\nu}_{e}$ spectrum is the signature of the inverted mass hierarchy and $\left|U_{e 3}\right|^{2}$ in the region I, irrespective of the solar neutrino solution.

(3) A soft $\bar{\nu}_{e}$ spectrum can appear only in the schemes with the SMA solution of the solar neutrino problem.

(4) The observation of any Earth matter effects will rule out the scenarios with the VO solution.

(5) The Earth matter effects in $\bar{\nu}_{e}$ spectrum will be the signature of the LMA solution.

(6) If the Earth matter effects are observed in the neutrino channels but not in the antineutrino channels, we either have the normal mass hierarchy with $\left|U_{e 3}\right|^{2}$ in region II or III, or the inverted mass hierarchy.

(7) If the Earth matter effects are significant in both the $\nu_{e}$ and $\bar{\nu}_{e}$ channels, the solar neutrino solution is LMA and $\left|U_{e 3}\right|^{2}$ lies in region II or III.

Let us now systematically consider all the possible combinations of the observations. In general, the "observations" consist of the following features of the spectra:

(i) The flavor of the neutronization peak can be either mixed $\left(\nu_{e}, \nu_{x}\right)$ or (almost) completely changed $\left(\nu_{x}\right)$.

(ii) The $\nu_{e}$ spectrum at the cooling stage can be either hard or composite.

(iii) The $\bar{\nu}_{e}$ spectrum can be of all possible types: soft (unchanged), hard, composite.

(iv) The Earth matter effects can be absent completely or observed in one of channels $\left(\nu_{e}\right.$ or $\left.\bar{\nu}_{e}\right)$, or observed in both channels.

The following conclusions can be drawn from the combinations of the above observations:

(A) Completely changed flavor $\left(\nu_{x}\right)$ of the neutronization peak, hard $\nu_{e}$ spectrum, and soft $\bar{\nu}_{e}$ spectrum. This configuration can be realized in only one case: normal hierarchy, SMA solution of the $\nu_{\odot}$ problem and $\left|U_{e 3}\right|^{2}$ in region I.
(B) Pure $\nu_{x}$-neutronization peak, hard $\nu_{e}$ spectrum, and composite $\bar{\nu}_{e}$ spectrum. This implies normal mass hierarchy, $\left|U_{e 3}\right|^{2}$ in region I, and two possible $\nu_{\odot}$ solutions, LMA or VO. The observation of Earth matter effects can distinguish between these two possibilities. For the LMA solution, the Earth effects will be present in the $\bar{\nu}_{e}$ channel and absent in the $\nu_{e}$ channel.

(C) Mixed $\left(\nu_{e}, \nu_{x}\right)$ flavor neutronization peak, composite $\nu_{e}$ spectrum, and soft $\bar{\nu}_{e}$ spectrum. This configuration can be realized in the three different cases: (i) SMA solution, normal mass hierarchy, and $\left|U_{e 3}\right|^{2}$ in region II, (ii) SMA solution, normal mass hierarchy, but $\left|U_{e 3}\right|^{2}$ in region III, (iii) SMA solution, inverted hierarchy, $\left|U_{e 3}\right|^{2}$ in region III. Clearly such a configuration is the signature of the SMA solution of the $\nu_{\odot}$ problem. The earth matter effect is expected to be weaker in the case (i).

(D) Mixed $\left(\nu_{e}, \nu_{x}\right)$ flavor neutronization peak, composite $\nu_{e}$ spectrum, composite $\bar{\nu}_{e}$ spectrum. This combination of observations can be realized in many cases: (i) SMA solution, inverted hierarchy, $\left|U_{e 3}\right|^{2}$ in region II, (ii)-(v) LMA solution, normal or inverted hierarchy, $\left|U_{e 3}\right|^{2}$ in regions II or III, (vi)-(ix) VO solution, normal or inverted hierarchy, $\left|U_{e 3}\right|^{2}$ in regions II or III. Studies of the Earth effects can give an important criterion for discriminating between the above cases: they are absent in the VO cases (vi)-(ix) and should be observable in the $\nu_{e}$ channel but not in $\bar{\nu}_{e}$ channel in the SMA case (i). In the LMA case, the Earth effects are expected both in the $\nu_{e}$ and $\bar{\nu}_{e}$ channels. Here a further discrimination can be done between (ii)-(v) by studying the degree of compositness: in the case of normal hierarchy one expects stronger effects in the neutrino channel.

(E) Mixed $\left(\nu_{e}, \nu_{x}\right)$ flavor neutronization peak, composite $\nu_{e}$ spectrum, hard $\bar{\nu}_{e}$ spectrum. Such a combination of observations is realized with inverted hierarchy, $\left|U_{e 3}\right|^{2}$ in region I, and SMA or LMA or VO solution. That is, the combination does not depend on the solution of the $\nu_{\odot}$ problem and turns out to be the signature of the inverted mass hierarchy. In the cases with SMA or LMA solutions the Earth effect can be observed in the $\nu_{e}$ channel only. No Earth effect is expected in the VO case.

No other combination of the observables is realized in the mixing scenarios under disussion.

Establishing the region in which $\left|U_{e 3}\right|^{2}$ lies is equivalant to giving bounds on the value of this mixing parameter, which correspond to the borders of the region. The borders depend on the precise density profile of the progenitor and can vary within a factor of $2-3$. Thus, from $\mathrm{SN}$ data we can get bounds on $\left|U_{e 3}\right|^{2}$ with an uncertainty of a factor of 2-3. In principle the value of $\left|U_{e 3}\right|^{2}$ can also be measured (again within a factor of 2-3) in the transition region where the effect (survival probability) depends substantially on $U_{e 3}$.

Observations (A), (B), and (E) above establish $\left|U_{e 3}\right|^{2}$ in region I, thus giving a lower bound of $\left|U_{e 3}\right|^{2} \geq 3 \times 10^{-4}$. Observations (C) and (D) establish $\left|U_{e 3}\right|^{2}$ to be in region II or III, thus giving an upper bound of $\left|U_{e 3}\right|^{2} \leqslant 3 \times 10^{-4}$. The upper bound obtained in the case of observations (C) and (D) is more than an order of magnitude stronger than the one the 
long baseline experiments are planning to achieve. The observation of the broadening of both the $\nu_{e}$ and $\bar{\nu}_{e}$ spectra, or significant Earth matter effects on both the $\nu_{e}$ and $\bar{\nu}_{e}$ spectra are sufficient to establish this bound.

A number of possible observations can rule out all the scenarios under discussion. The observation of (a) a pure $\nu_{e}$ neutronization peak and (b) a soft $\nu_{e}$ spectrum during the cooling stage is not possible with any of these neutrino mass spectra. The pairs of observations (a) pure $\nu_{x}$ neutronization peak and a composite $\nu_{e}$ spectrum, (b) mixed flavor neutronization peak and a pure hard $\nu_{e}$ spectrum, and (c) a hard spectrum for both $\nu_{e}$ and $\bar{\nu}_{e}$ are also fatal for all the scenarios.

The final neutrino spectra can thus help in resolving three kinds of ambiguities that remain to be resolved with the current data: (i) the solution of the solar neutrino problem, (ii) the type of mass hierarchy ( $\operatorname{sign}$ of $\Delta m^{2}$ ), and (iii) the value of $\left|U_{e 3}\right|^{2}$.

The difference in the "temperatures" of the original $\nu_{e}$, $\bar{\nu}_{e}$ and $\nu_{x}$ spectra is a crucial issue as far as the observations are concerned. In this article we have given the expressions for the final spectra in terms of undetermined values of these temperatures, and have given the qualitative features of the final spectra. The data should eventually be analyzed in terms of not only the oscillation parameters, but also the temperatures as free parameters. This will lead to the determination of temperatures of the spectra directly from the data. If a substantial temperature hierarchy is present within the $\left(\nu_{e}-\nu_{x}\right)$ or $\left(\bar{\nu}_{e}-\nu_{x}\right)$ pair, the effects of broadening of the spectra and the Earth matter effects may be more pronounced.
The implications of results of this paper will depend on when neutrino burst from the Galactic supernova will be detected.

A number of results can be obtained from other experiments unrelated to the supernova neutrinos. Clearly, all the schemes considered above will be rejected if sterile neutrinos will be discovered in the oscillations of solar or atmospheric neutrinos, or if the LSND result will be confirmed. In this case, one will be forced to consider the schemes with sterile neutrinos [9].

On the other hand, there is a good chance that within several years existing and future experiments will allow us to identify one of the solutions of the solar neutrino problem considered in this paper. This will significantly diminish the number of possible schemes and will allow us to further sharpen the predictions of the effects for supernova neutrinos.

The purpose of this paper was to indicate the effects which can in principle testify for neutrino conversions and various features of the neutrino mass and flavor spectra. Clearly, further detailed studies are needed for specific detectors to clarify the detectability of the discussed effects and to conclude how far we can go in the program of identification of the neutrino mass spectrum.

\section{ACKNOWLEDGMENTS}

One of us (A.D.) would like to thank K. R. S. Balaji, J. Beacom, J. Lattimer, G. Raffelt and K. Uryu for discussions and useful comments. He would also like to thank The Abdus Salam ICTP, where most of this work was completed.
[1] SuperKamiokande Collaboation, Y. Fukuda et al., Phys. Rev. Lett. 82, 2644 (1999); SuperKamiokande Collaboration, K. Scholberg hep-ex/9905016.

[2] SOUDAN 2 Collaboration, E. Peterson, Nucl. Phys. B (Proc. Suppl.) 77, 111 (1999).

[3] MACRO Collaboration, talk by M. Spurio at the 6th Topical Seminar on Neutrino and AstroParticle Physics, San Miniato, Italy, 1999, hep-ex/9908066.

[4] CHOOZ Collaboration, M. Apollonio et al., Phys. Lett. B 466, 415 (1999).

[5] For global fits to the solar neutrino data, see e.g. J. N. Bahcall, P. I. Krastev and A. Yu. Smirnov, Phys. Rev. D 58, 096016 (1998); P. I. Krastev, hep-ph/9905458; M. C. GonzalezGarcia, P. C. de Holanda, C. Pena-Garay and J. W. F. Valle, Nucl. Phys. B573, 3 (2000).

[6] G. L. Fogli, E. Lisi, D. Montanino and A. Palazzo, Phys. Rev. D 61, 073009 (2000).

[7] LSND Collaboration, D. White, Nucl. Phys. B (Proc. Suppl.) 77, 207 (1999).

[8] K. Eitel, New J. Phys. 2, 1 (2000).

[9] A. S. Dighe and A. Yu. Smirnov (in preparation).

[10] L. Wolfenstein, Phys. Rev. D 17, 2369 (1978); 20, 2634 (1979); S. P. Mikheyev and A. Yu. Smirnov, Zh. Éksp. Fiz. 91,
7 (1986) [Sov. Phys. JETP 64, 4 (1986)]; Yad. Fiz. 42, 1441 (1985) [Sov. J. Nucl. Phys. 42, 913 (1986)]; in Proceedings of the 6th Moriond Workshop on Massive Neutrinos in Astrophysics and Particle Physics, Tignes, France, 1986, edited by O. Fackler and J. Tran Than Van, p. 355; G. M. Fuller et al., Astrophys. J. 322, 795 (1987); G. M. Fuller et al., ibid. 389, 517 (1992); G. Raffelt and G. Sigl, Astropart. Phys. 1, 165 (1993).

[11] J. Arafune et al., Phys. Rev. Lett. 59, 1864 (1987); J. Arafune et al., Phys. Lett. B 194, 477 (1987); P. O. Lagage et al., ibid. 193, 127 (1987); H. Minakata et al., Mod. Phys. Lett. A 2, 827 (1987); S. P. Rosen, Phys. Rev. D 37, 1682 (1988); H. Minakata and H. Nunokawa, ibid. 38, 3605 (1988).

[12] D. Nötzold, Phys. Lett. B 196, 315 (1987).

[13] P. Reinartz and L. Stodolsky, Z. Phys. C 27, 507 (1985).

[14] A. E. Chudakov, O. G. Ryazhskaya and G. T. Zatsperin, talk given at the Denver 1973 Cosmic Ray Conference, 1973, Vol. 3, p. 2007; S. Choubey, D. Majumdar and K. Kar, J. Phys. G 25, 1001 (1999); D. Majumdar and A. Raychaudhuri, Phys. Rev. D 60, 053001 (1999).

[15] H. Athar, J. T. Peltoniemi and A. Yu. Smirnov, Phys. Rev. D 51, 6647 (1995).

[16] K. Fujikawa and R. E. Shrock, Phys. Rev. Lett. 45, 963 (1980). 
[17] C.-S. Lim and W. J. Marciano, Phys. Rev. D 37, 1368 (1988); E. Kh. Akhmedov and Z. G. Berezhiani, in Les Arcs 1990, Proceedings, Particle Astrophysics, p. 305; Nucl. Phys. B373, 479 (1992).

[18] G. C. McLaughlin, J. M. Fetter, A. B. Balantekin and G. M. Fuller, Phys. Rev. C 59, 2873 (1999).

[19] A. Yu. Smirnov, D. N. Spergel and J. N. Bahcall, Phys. Rev. D 49, 1389 (1994).

[20] Kamiokande Collaboration, K. S. Hirata et al., Phys. Rev. Lett. 58, 1490 (1987); IMB Collaboration, R. M. Bionta et al., ibid. 58, 1494 (1987); E. N. Alexeyev et al., Pis'ma Zh. Éksp. Teor. Fiz. 45461 (1987) [JETP Lett. 45, 589 (1987).

[21] L. M. Krauss, Nature (London) 329, 689 (1987); J. A. Frieman, H. E. Haber and K. Freese, Phys. Lett. B 200, 115 (1988); A. E. Chudakov, Ya. S. Elensky and S. P. Mikheyev, in Cosmic Gamma Rays, Neutrinos, and Related Astrophysics, edited by M. M. Shapiro and J. P. Wefel (NATO Advanced Study Institute, Series C: Mathematical and Physical Sciences, Vol. 270) (Kluwer Academic, Dordrecht, 1989), p. 131; B. Jegerlehner, F. Neubig and G. Raffelt, Phys. Rev. D 54, 1194 (1996).

[22] T. P. Walker and D. N. Schramm, Phys. Lett. B 195, 331 (1987).

[23] S. P. Mikheev and A. Yu. Smirnov, Pis'ma Zh. Éksp. Teor. Fiz. 46, 11 (1987) [JETP Lett. 46, 10 (1987)]; Usp. Fiz. Nauk. 153, 3 (1987) [Sov. Phys. Usp. 30, 759 (1987)].

[24] T. K. Kuo and J. Pantaleone, Phys. Rev. D 37, 298 (1988).

[25] S. P. Mikheev and A. Yu. Smirnov, Prog. Part. Nucl. Phys. 23, 41 (1989).

[26] T. K. Kuo and J. Pantaleone, Rev. Mod. Phys. 61, 937 (1989).

[27] F. Buccella, S. Esposito, C. Gualdi and G. Miele, Z. Phys. C 73, 633 (1997).

[28] A. Yu. Smirnov, talk given at Symposium on New Era in Neutrino Physics, Tokyo, hep-ph/9811296.

[29] A. S. Dighe, in Valencia 99, International Workshop on particle Astrophysics and Cosmology: From Theory to Observation, 1999 [Nucl. Phys. B. (Proc. Suppl.) 81, 294 (2000)].

[30] G. Dutta, D. Indumathi, M. V. N. Murthy and G. Rajasekaran, Phys. Rev. D 61, 013009 (2000).

[31] D. O. Caldwell, G. M. Fuller and Y. Z. Qian, Phys. Rev. D (to be published), astro-ph/9910175.

[32] For a comprehensive review of the astrophysical aspects of a type II supernova burst, see H. Suzuki, in Physics and Astrophysics of Neutrinos, edited by M. Fukugita and A. Suzuki (Springer-Verlag, Berlin, 1994), p. 763.

[33] C. J. Horowitz and G. Li, Phys. Lett. B 443, 58 (1998); S. Hannestad et al. astro-ph/9912242.

[34] E. S. Myra, J. M. Lattimer and A. Yahil, in Supernova 1987A in the Large Magellanic Cloud, edited by M. Kafatos and A. Michalitsianos (Cambridge University Press, Cambridge, England, 1988).

[35] R. Mayle, J. R. Wilson and D. N. Schramm, Astrophys. J. 318, 288 (1987).

[36] H.-T. Janka and W. Hillenbrandt, Astron. Astrophys., Suppl. Ser. 78, 375 (1989); H.-T. Janka and W. Hillenbrandt, Astron. Astrophys. 224, 49 (1989).
[37] G. Raffelt, Stars as Laboratories of Fundamental Physics (The University of Chicago Press, Chicago, 1996).

[38] P. F. Harrison, D. H. Perkins and W. G. Scott, Phys. Lett. B 396, 186 (1997); 458, 79 (1999).

[39] S.-H. Chiu and T. K. Kuo, Phys. Rev. D 61, 073015 (2000).

[40] J. R. Wilson et al., Ann. (N.Y.) Acad. Sci. 470, 267 (1986).

[41] R. Mayle and J. R. Wilson, The Nuclear Equation of State, NATO Conference Proceedings (Springer, Berlin, 1989); S. A. Colgate, Nature (London) 341, 489 (1989).

[42] G. E. Brown, H. A. Bethe and G. Baym, Nucl. Phys. A375, 481 (1982).

[43] L. Landau, Phys. Z. Sowjetunion 2, 46 (1932); C. Zener, Proc. R. Soc. London A137, 696 (1932).

[44] S. P. Mikheyev and A. Yu. Smirnov, Yad. Fiz. 42, 1441 (1985) [Sov. J. Nucl. Phys. 42, 913 (1985)].

[45] T. K. Kuo and J. Pantaleone, Phys. Rev. D 39, 1930 (1989).

[46] MINOS Technical Design Report No. NuMI-L-337 TDR, 1998.

[47] T. K. Kuo and J. Pantaleone, Phys. Rev. Lett. 57, 1805 (1986).

[48] F. J. Botella, C. S. Lim and W. J. Marciano, Phys. Rev. D 35, 896 (1987).

[49] H.-T. Janka, Astropart. Phys. 3, 377 (1995).

[50] S. P. Mikheyev and A. Yu. Smirnov, in Moriond 87, Proceedings of the 7th Moriond Workshop on New and Exotic phenomena, Les Arcs, France, 1987, edited by O. Fackler and J. Trân Thanh Vân (Frontières, Paris, 1987), p. 405; A. J. Baltz and J. Weneser, Phys. Rev. D 50, 5971 (1994); 51, 3960 (1994); P. I. Krastev, hep-ph/9610339; Q. Y. Liu, M. Maris and S. T. Petcov, Phys. Rev. D 56, 5991 (1997); J. N. Bahcall and P. I. Krastev, Phys. Rev. C 56, 2839 (1997); M. Maris and S. T. Petcov, Phys. Rev. D 56, 7444 (1997).

[51] E. Kh. Akhmedov, Nucl. Phys. B538, 25 (1999); E. Kh. Akhmedov, A. Dighe, P. Lipari and A. Yu. Smirnov, Nucl. Phys. B542, 3 (1999).

[52] F. Vissani, hep-ph/9708483; V. Barger et al., Phys. Lett. B 437, 107 (1998); A. Baltz, A. S. Goldhaber and M. Goldhaber, Phys. Rev. Lett. 81, 5730 (1998); H. Georgi and S. L. Glashow, hep-ph/9808293.

[53] T. Totani, K. Sato, H. E. Dalhed and J. R. Wilson, Astrophys. J. 496, 216 (1988).

[54] A. Burrows, D. Klein and R. Gandhi, Phys. Rev. D 45, 3361 (1992); Nucl. Phys. B (Proc. Suppl.) 31, 408 (1993).

[55] G. M. Fuller, W. C. Haxton and G. C. McLaughlin, Phys. Rev. D 59, 085005 (1999) and references therein.

[56] O. G. Ryazheskaya, V. G. Ryasny and O. Saavedra, Pis'ma Zh. Éksp. Teor. Fiz. 56, 433 (1992) [JETP Lett. 56, 417 (1992)]; Nuovo Cimento A 106, 257 (1993).

[57] SNO Collaboration, J. Boger et al., nucl-ex/9910016.

[58] LVD Collaboration, M. Aglietta et al., Nuovo Cimento C 18, 629 (1995).

[59] MACRO Collaboration, M. Ambrosio et al., Astropart. Phys. 8, 123 (1998).

[60] J. F. Beacom and P. Vogel, Phys. Rev. D 60, 033007 (1999); P. Vogel, astro-ph/9904338; J. F. Beacom, hep-ph/9909231.

[61] CHOOZ Collaboration, M. Apollonio et al., Phys. Rev. D 61, 012001 (2000).

[62] S. Choubey and K. Kar, Phys. Rev. D 61, 097301 (2000). 